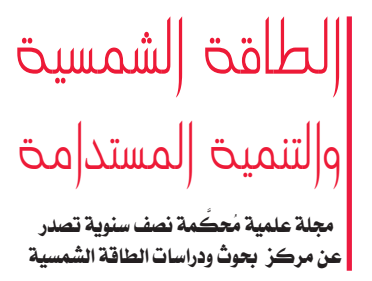

\title{
الاستثمار في الطاقات المتجددة كآلية لرفع معدلات النمو الاقتصادي للدول العربية: \\ دراسة قياسية مقارنة بين الدول النفطية وغير النفطية \\ زرزار العياشي ${ }^{1}$ و مداحي محمد 2 \\ 1 جامعت 20 أوت 1955 - سكيكدة. الجزائر. 'جامعت البويرة. الجزائر \\ بريد إلكتروني:Zerzar_18@yahoo.fr'1,meddahi26@gmail.com²
}

الملخص: الهلدف من هذه الورقت البحثيت هو دراست ديناميكيت الطاقات المتجددة يِّ الدول العربيت باستخدام الأدوات

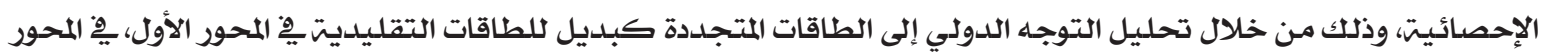

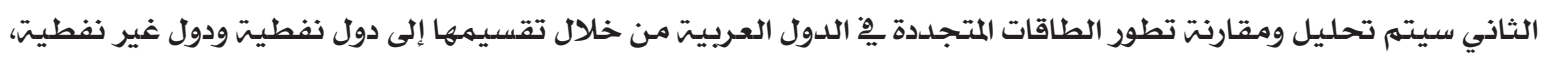

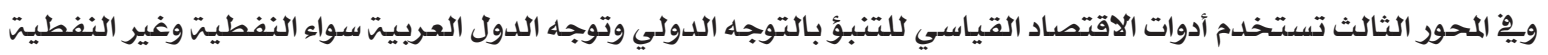

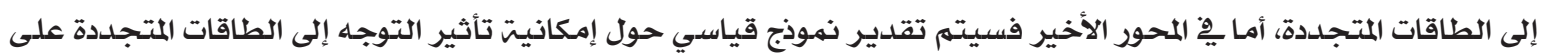

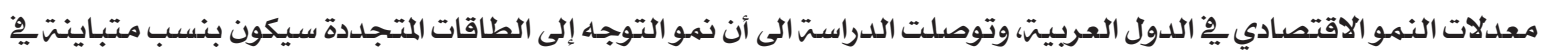

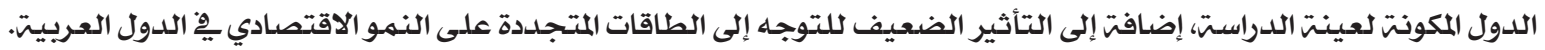
كلمات استدلاليت: الطاقات المتجددة، الطاقات التقليديت، النمو الاقتصادي، البلدان العربيت، الإنتاج.

Investment in Renewable Energy as a Tool to Increase the Economic Growth of the Arab Countries: A Comparative Standard Study between Oil and Non-Oil Producing Countries

\author{
Zerzar layachi ${ }^{1}$, and Medahi mouhamed ${ }^{2}$ \\ ${ }^{1}$ University of 20 Aaut 1955, Skikda, Algeria. ${ }^{2}$ University of Bouira, Algeria.
}

Abstract: The aim of this paper is to study the dynamics of renewable energies in the Arab countries by using statistical tools through the analysis of the international move to renewable energies as an alternative to the traditional energies in the first axis.

The second part will analyze and compare the evolution of renewable energies in the Arab countries through dividing them into oil producing countries and non- oil producing ones .In the third axis, tools of econometrics are used to predict the international trend and that of the Arab countries, both oil and non-oil to renewable energy. The last part estimates the standard model about the possible impact of moving energies on economic growth rates in the Arab countries. The study concluded that the growing renewable energies will be different in the constituent countries of the study sample, in addition to the weak impact of the move towards renewable energies on economic growth in the Arab countries. 
Keywords: Renewable energy, traditional energy, economic growth , the Arab countries, prodiction .

محاور الدراستة: لمعالجت الموضوع سوف نتطرق إلى العناصر

التاليت:

المحور الأول: التأصيل النظري لمفهوم الطاقات المتجدددة.

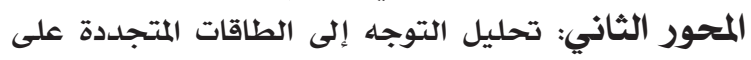
المستوى الدولي. المحور الثالث: تحليل توجه الدول العربيت إلى الطاقات المتجدةد. المحور الرابع: التنبؤ بتوجه الدول العربيت إلى الطاقات المتجددة خلال الفترة 2010-2016. المحور الخامس: أثر التوجه إلى الطاقات المتجدة المدادة على معدلات النمو الاقتصادي. اتخر التوجل

\section{المحور الأول: الطاقات المتجددة تأصيل نظري}

1. الإطار النظري للطاقات المتجددة: عندما عرف الإنسان النار،

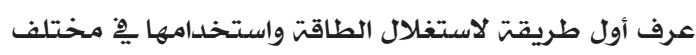
أغراضه الحياتيتة، مثل: طهي الطعام وتدفئت الكهف وإنارة

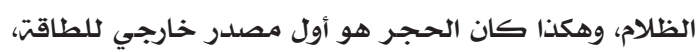
ثم تالاه الخشب وغيره من أدوات إثعال النار، والحصول على اولى مهار الطاقت الحراريتي.

1-1 مفهوم الطاقت المتجددة ومصدادرها: لقد تزايد الاهتمام العالمي حاليا بتنويع وتجديد مصادر الطاقت وخاصت المصادر

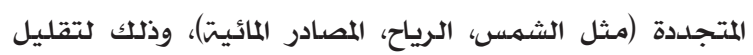

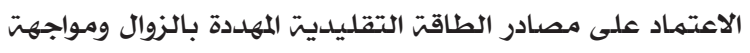
التهديدات البيئيت (تزايد معدلات الانبعاثات الحراريت) للتغير

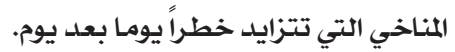
وبالربط بين إنتاج الطاقت وتلوث البيئت وما نتج عن ذلك من دعوات إقليميت وعالميت لضرورة التعامل مـع المشاكل البيئيت بصورة أكثر فاعليت، وتعالي الصرخات من ظاهرة الاحتباس الحراري التي سببها ارتفاع نسبت غاز ثاني أكسيد الكربون الناتج عن زيادة حرق الوقود الأحفوري يِّ محطات الكهرباء وعوادم السيارات والمصانع وحرائق الغابات، مما أدى الى الزيادة درجت

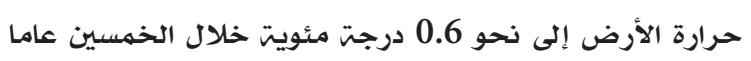
الماضيت، وهو ما انعكس على زيادة معدل ذوبان الجليد يِّ القطب الشمالي، ومن ثم ارتفاع منسوب المياه يِّ البحار والمحيطات لتهلدد يِّ النهايت أماكن كثيرة وشاسعت من الأرض، نجد الطاقت تشارك بنحو 24\% من غازات الاحتباس الحراري مما يضع

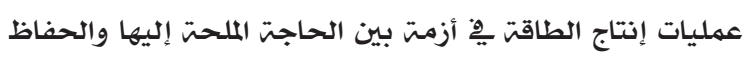
على البيئتة. وتتميز مصادر الطاقت المتجددة بقابليت استغلالها
الإقدمة

تشكل كل من الطاقات المتجددة والطاقت النوويت

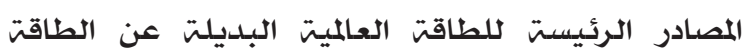
الأحفوريت، وهناك اهتمام عالمي كبير بهذين المصدرين

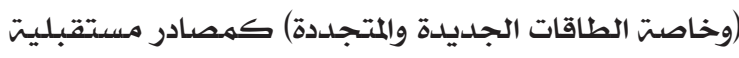
للطاقت بحيث تكون بديلا للطاقت الأحفوريت التي تسعى

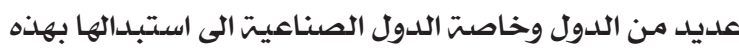

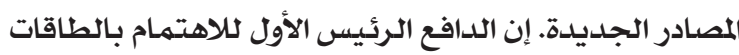

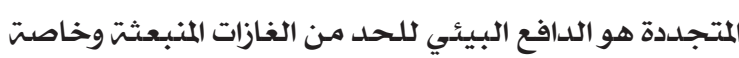

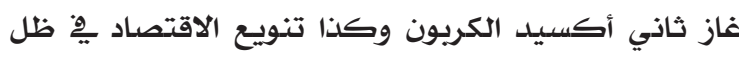

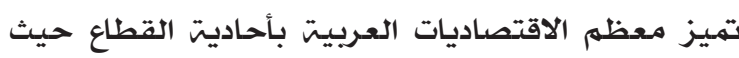

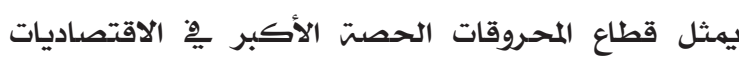
العربيت النفطيت.

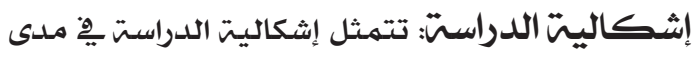
توجه الدول العربيت قيد الدراست إلى الطاقات المتجددة

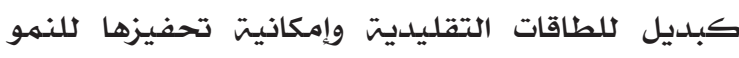
الاقتصادي باستخدام أدوات القياس الاقتصادي. عينتم الدراست: ستجرىى الدراست على الدول العربيت

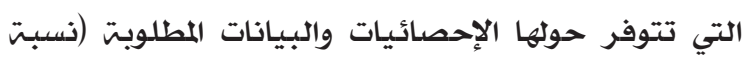
الطاقات المتجددة من إجمالي استهالاك الطاقتة، النهو الاقتصادي للدول مهثلا بنمو الناتج المحلي الإجمالي) وستكون فترة الدراست محصورة بين سنتي 1995 - 2009

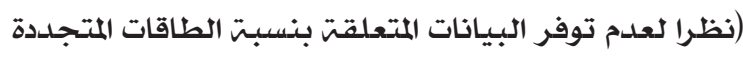
من إجمالي استهالك الطاقت يْ سنتي 2010 و و2011 ويِ دول أخرى خلال سنت 2011 وبالتالي لا يمكن تطبيق نهاذج معطيات بانل يِّ حالت فقدان مشاهدة أو مشاهدتين وتم حذف المثاهدات الناقصت من كل الدول وتم تحديد سنت

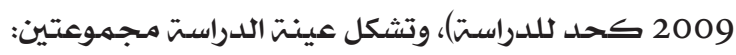
ـ المجموعت الأولى تمثل الدول النفطيت وهي: الجزائر،

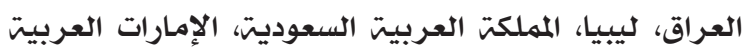
المتحدة. ـ المجموعت الثانيت تمثل الدول غير النفطيت وهي: مصر، لبنان، المغرب، السودان، سوريا، تونس، اليهن. فرضيت الدراستّ: كإجابت مبدئيت على المشكلت المطروحت يمكن وضع الفرضيت التاليت:

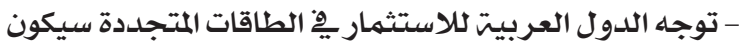

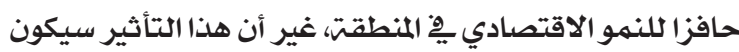

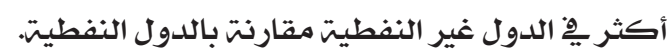


المياه الذي يهكن أن يصل إلى فرق 10 درجات مئويت. 2-1-1 - طاقت الكتلت الحيويت: تكمن أهميت طاقت الكتلت الحيويت إن فرقيت

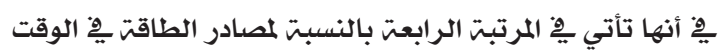

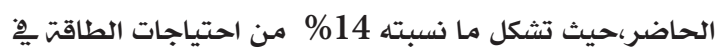
العالم، وتزداد أهميت هذه الطاقت يِّ الدول الناميتّ حيث ترتفع

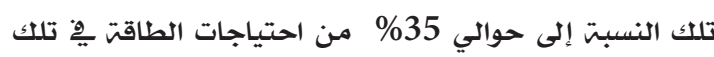

$$
\text { الدول، وخاصت يِّ المناطق الريفيت [7]. }
$$

إن طاقت الكتلت الحيويت أو كما تسهى أحيانا الطاقت الحيويت

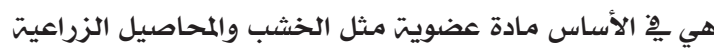
والمخلفات الحيوانيت، وهذه الطاقت هي طاقت متجددة لأنها تحول طاقت الشمس إلى طاقت مخزنت ِِّ النباتات عن طريق عمليت التمثيل الضوئي، فطالما هناك نباتات خضراء فهناك فركاك طاقت شمسيت مخزنت فيها، وبالتالي لدينا طاقت الكتلت الحيويت التي نستطيع الحصول عليها بطرق مختلفت من هذه فئه النباتات. أما مصادر الكتلت الحيويت ِِّ الوقت الحاضر فهي:

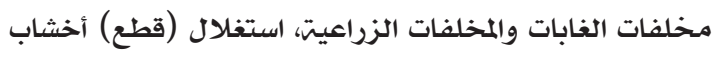
الغابات بشكل مدروس، وفضلات المدن، والمحاصيل التي تزرع خصيصا لغايات الحصول على الطاقت منها. والطاقت الحيويت هي الطاقت التي تستمد من المواد العضويت كإحراق النباتات وعظام ومخلفات الحيوانات والنفايات والمخلفات الزراعيت، والنباتات المستخدمت فِ إنتاج طاقت الكتلت الحيويت يمكن

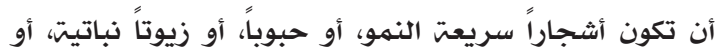

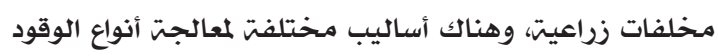
الحيوي. ويعطي كل أسلوب من الأساليب السابقت منتجاته الخاصت به مثل غاز الميثان والكحول والبخار والأسمدة الكيماويت، ويعد غاز الإيثانول واحداً من أفضل أنواع الوقود المستخلصت من الكتلت الحيويت وهو يستخرج بشكل رئيس من محاصيل الذرة وقصب السكر.

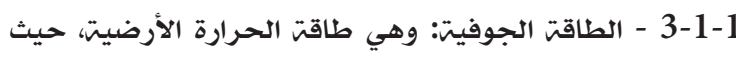
يُستفاد من ارتفاع درجت الحرارة يِّ جوف الأرض باستخراج

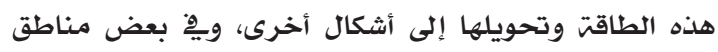
الصلدوع والتشققات الأرضيت تتسرب المياه الجوفيت عبر الصدوع والثقوق إلى أعماق كبيرة بحيث تلامس مناطق شديدة السخونت فتسخن وتصعد إلى أعلى فوارة ساخنتة، وبعض هذه الينابيع يثور ويهمد عدة مرات يِّ الساعت وبعضها

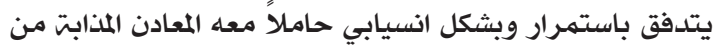
طبقات الصخور العميقت، ويظهر بذلك ما يطلق عليه الينابيع الحارة، ويقصد الناس هذا النوع من الينابيع للاستشفاء، بالإضافت إلى أن هناك مشاريع تقوم على استغلال حرارة المياه المنطلقت من الأرض فِّ توليد الكهرباء.
المستمر دون أن يؤدي ذلك إلى استنفاد منبعها، فالطاقت المتجددة هي تلك الموارد التي نحصل عليها من خلال تيارات الطاقت التي

يتكرر وجودها يِّ الطبيعت على نحو تلقائي ودوري [1]. كذلك نعني ”بالطاقت المتجددة، الكهرباء التي يتم توليدها

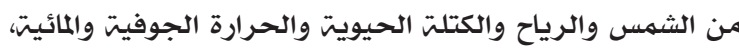
وكذلك الوقود الحيوي والهيدروجين المستخرج من المصادر المتجددة [2] . أو هي تلك المصادر الطبيعيت غير الناضبت والمتوفرة مِّ الطبيعت سواء كانت محلدودة أو غير محلدودة إلا أنها متجددة،

وهي نظيفت لا ينتج عن استخدامها تلوث بيئي [3].

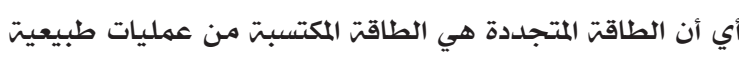
تتجدد باستمرار [4] · وبالتالي فهي عبارة عن مصادر طبيعيت دائمت وغير ناضبت ومتوفرة يِّ الطبيعت سواء أكانت محلدودة أو غير محدودة ولكنها متجددة باستمرار، وهي نظيفت لا ينتج عن استخدامها تلوث بيئي نسبيا، ومن أهم هذه المصادر الطاقت

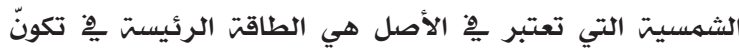
مصادر الطاقت وكذلك طاقت الرياح وطاقت المد والجزر والأمواج [5]. فأهم أثكال ومصادر الطاقت المتجددة تتمثل يِّ: 1-1-1. طاقت المياه: يعود تاريخ الاعتماد على المياه كمصدر آلمدرد للطاقت إلى ما قبل اكتشاف الطاقت البخاريت فِّ القرن الثامن عشر، حتى ذلك الوقت،كان الإنسان يستخدم مياه الأنهار پِّ تشغيل بعض النواعير التي كانت تستعمل لإدارة

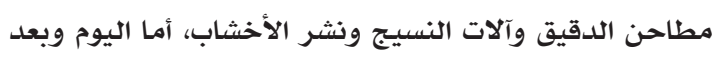
أن دخل الإنسان عصر الكهرباء، بدأ استعمال المياه لتوليد الطاقت الكهربائيت كما نشهد يِّ دول عديدة مثل النرويج والسويد وكندا والبرازيل، ومن أجل هذه الغايت، تقام محطات توليد الطاقت على مساقط الأنهار، وتبنى السدود والبحيرات الاصطناعيت لتوفير كميات كبيرة من الماء تضمن تشغيل

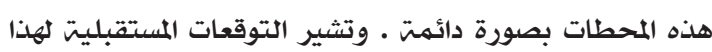
المصدر من الطاقت إلى زيادة تقدر بخمست أضعاف الطاقت الحاليت بحلول عام 2020 [6] .

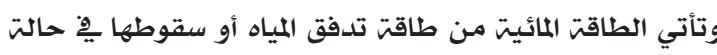

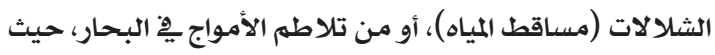
تنشأ الأمواج نتيجت لحركت الرياح وفعلها على مياه البحار والمحيطات والبحيرات، ومن حركت الأمواج هذه تنشأ طاقت يمكن استغلالها وتحويلها إلى طاقت كهربائيتة، حيث تنتج الأمواج هِ الأحوال العاديت طاقت تقدر ما بين 10 إلى 100 كيلو وات لكل متر من الشاطئ يِّ المناطق متوسطت البعد عن

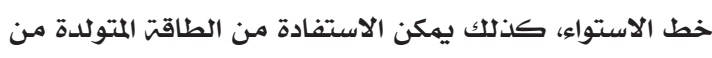

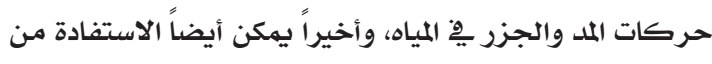
الفارق ِِّ درجات الحرارة بين الطبقتين العليا والسفلى من 
هذه الطاقت مستقبلا يِّ تحليت المياه وإنتاج الكهرباء بشكل واسع، وتعتبر الطاقت الفولتيت الضوئيت الشمسيت صناعت عالميت

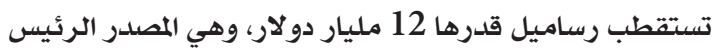
للطاقت المتجددة التي يتهم توزيعها فعليًا (المستهلكون الذين

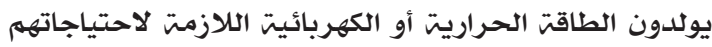

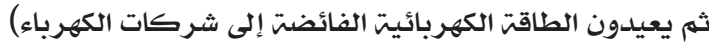

1-2 - خصائص مصادر الطاقت البديلت: المقصود بالمصادر الحاليت للطاقت تلك المصادر التي تزود البشر بالجزء الأساسي والأكبر

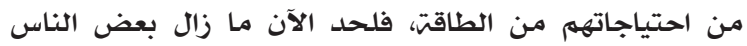
يعتمدون على أخشاب الأشجار فِ تلبيت جزء من من منطلباتهم من الطاقت، كما أن بعضهم الآخر مازال يعتمد على الحيوانات يْ التنقل وحمل الحاجيات والحراثت، ونجد بعضهم يستخدم مصادر الطاقت المتجددة كالطاقت الشمسيت والهوائيت للحصول على بعض متطلباته من الطاقتة، إلا أن هذه المصادر مجتمعتص ليست ذات قيمت كميت تذكر بالمقارنت مـع ما يستهلكه الإنسان

$$
\text { من مصادر أخرى [10]. }
$$

وإن خصائص المصادر الطاقت المتجددة وطبيعتها عموما تفرض المرض تمان على الإنسان تطوير التكنولوجيا الملائمت لاستغلالها، ويتضح هذا ولاديا

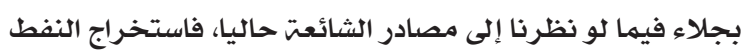
مثثلا فرض على الإنسان تطوير تكنولوجيا الحفر، وأهم هذه

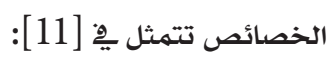

إن مصادر الطاقت البديلت المرشحت لأن تلعب دورا هاما ِِيِ حياة الإنسان وأن تساهم فِ تلبيتة نسبت عاليت من متطلباته من الطاقت هي مصادر دائمتم طويلت الأجل ذلك لأنها مرتبطت أساسا بالشمس والطاقت الصادرة عنها. إن مصادر الطاقت البديلت رغم ديهومتها على المدى البعيد فانها لا تتوفر بشكل منتظم طول الوقت وعلى مدار الساعتة، فهي ليست مخززونا جاهزا نستعمل منـه ما نشاء متى نشاء فمصادر الطاقت البديلت تتوفر أو تختفي بشكل خارج قدرة الإنسان على التحكم فيها أو تحديد مقادير المتوفر منها، كالشمس وشدة الإثعاع. إن شدة الطاقتة يْ المصادر البديلت ليست عاليت التركيز، وبالتالي فإن استخدلام هذه المصادر يتطلب استعمـال العديد من الأجهزة ذات المساحات والأحجام الكبيرة، والواقع أن هذا هو أحد أسباب ارتفاع التكلفت الأولويت لأجهزة الطاقت البديلت وهو ما يشكل ِِّ نفس الوقت أحد عوائق انتشارها

$$
\text { السـريع. }
$$

تتوفر أشكال مختلفت من الطاقت ٍِِ مصدادر الطاقت البديلت
4-1-1 - طاقت الرياح: وهي الطاقت المتولدة من تحريك ألواح كبيرة مثبتت بأماكن مرتفعت بفعل الهواء، ويتم إنتاج الطاقت

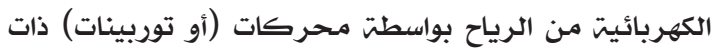
ثلاثت أذرع دوَّارة تحمل على عمود تعمل على تحوياح بواسطى محريل الطاقت الحركيت للرياح إلى طاقت كهربائيت، فعندما تمر الرياح

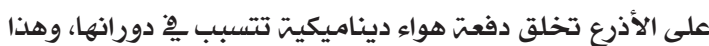
الدوران يشغل التوربينات فتنتج طاقت كهربائيت، وبتعبير آخر فان الطاقت الهوائيت هي الطاقت المستمدة من حركت الهواء والرياح، واستخدمت طاقت الرياح منذ أقدم العصور، سواء ِِّ تسيير السفن الشراعيت، وإدارة طواحين الهواء لطحن الغلال

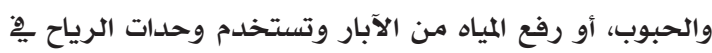
تحويل طاقت الرياح إلى طاقت ميكانيكيت تستخدم مباشرة أو يتم تحويلها إلى طاقت كهربائيت من خلال مولدات [8].

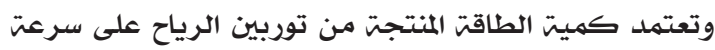

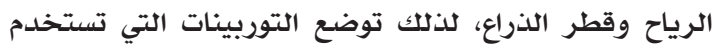

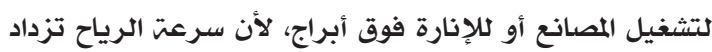

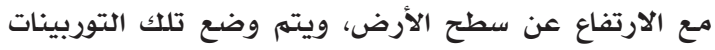
بأعداد كبيرة على مساحات واسعت من الأرض لإنتاج أكبر

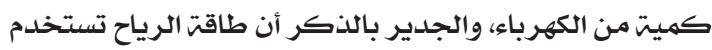

$$
\text { كذلك بِ تسيير المراكب والسفن الشراعيت. }
$$

1-1-1 - الطاقت الشمسيت: تعد الشمس من أكبر مصادر الضوء والحرارة الموجودة على وجه الأرض، وتتوزع هذه الطاقت-

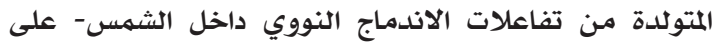

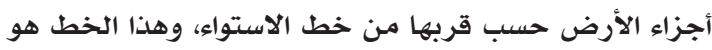
المنطقت التي تحظى بأكبر نصيب من تلك الطاقت، والطاقت الحراريت المتولدة عن أشعت الشمس يُستقاد منها عبر تحويلها إلى (طاقت كهربائيت) بواسطت (الخلايا الشمسيت). وهناك طريقتان لتجميع الطاقت الشمسيت، الأولى: بأن يتم تركيز أثعت الشمس على مجمع بواسطت مرايا محدبت الشكل، ويتكون المجمع عادة من عدد من الأنابيب بها ماء أو هواء، حيث تسخن حرارة الشمس الهواء أو تحول الماء إلى بخار.

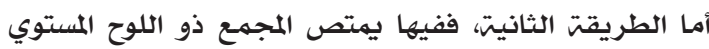
حرارة الشمس، وتستخدم الحرارة لتنتج هواء ساخنا أو بخارا. إن استخدام الشمس كمصدر للطاقت هو من بين المصادر البديلت للنفط التي تعقد عليها الآمال المستقبليت لكونها طاقت نظيفت لا تنضب، لذلك نجد دولا عديدة تهتم بتطوير هذا المصدر وتضعه هدفا تسعى لتحقيقه، وتستخلدم الطاقت الشمسيت حاليا ِِ2 تسخين المياه المنزليت وبرك السباحت والتدفئت والتبريد كما يجري يِّ أوروبا وأمريكا، أما يِّ دول العالم الثالث فتستعمل لتحريك مضخات المياه ِِِ المناطق الصحراويت الجافتة، وتجري الآن محاولات جادة لاستعمال 
غياب التشريعات والسياسات للاستثمارات التي من شأنها أن تحقق أهداف تنميت مصادر الطاقت المتجددة، والقوانين التي من شأنها

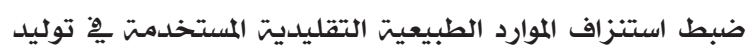

الطاقت:.

غياب التنظيم والتنسيق المؤسسي على المستوى الوطني والإقليمي

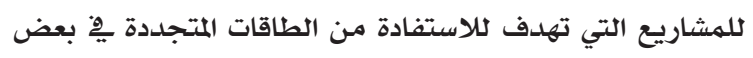

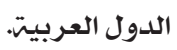

عدم بروز دور الحكومات يِّ تعزيز وترسيخ استخدام تقنيات

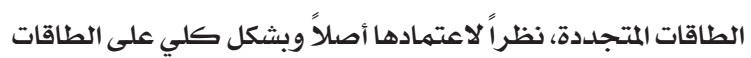
التقليديت ِِّ مشاريعها المختلفت. غياب البرامج التوعويت للمواطنين المبنيت على أسس علميت

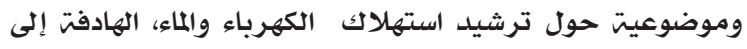
إحلال الطاقات المتجددة مكان استخدامات الطاقت التقليديت. ضعف دور القطاع الخاص يِّ نشر تقنيات الطاقات المتجددة، المتمثل ِِّ عدم استثماره ِِِ مجال إنشاء مصانع الصناعات الخفيفت القائمت على تجميع وتركيب الأجهزة التي توظف

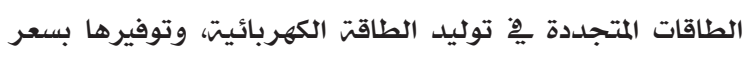
معقول للمستهلك. ندرة المشروعات التي تهدف إلى توظيف الطاقات المتجددة ِِيِ

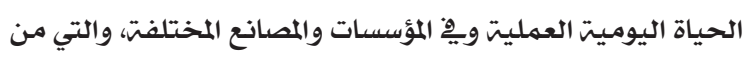
شأنها أن تقلل من التلوث البيئي الناجم عن استخدامـات الطاقات التوات التقليديت.

ندرة بعض أنواع الطاقات المتجددة كالمياه وعدم ثبات البعض الباد الآخر كالرياح يحول دون استثمارها، هذا إلى جانب ارتفاع تكلفت استخدام بدائل الطاقات التقليديت حالياً.

\section{المحور الثاني: تحليل التوجه إلى الطاقات التجددة على المستوى اللدولي}

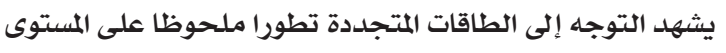
الدولي إذا أخذناه بالقيم الجاريتة، إلا أن نسبت هذا التوجه إلى إلى إجمالي الطاقت يِّ انخفاض مستمر منذ سنت 1971 إلى سنت 2010 خاصت الفترة 2008-2010، ومن خلال الشكل 1 يلاحظ أن معدل نهو حصت الطاقات المتجددة على المستوى الدولي بلغ

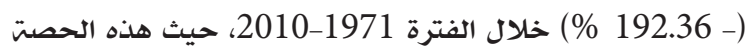
تشكل نسبت 12.68 \% سنت 1971 ـِ حين أصبحت ثمثل نسبت 4.70 \% سنت 2010. إن نتائج البيان السابق لا تعطينا أسباب واضحت عن الاتجاه السالب لنسبت الطاقات المتجددة إلى إجمالي الطاقتة، وعند تحليل جغرافيت التوجه إلى الطاقت المتجددة يتضح لنا جليا أن الدول

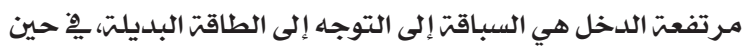
أن الدول منخفضت ومتوسطت الدخل تشهد معدلات متناقصت
الأمر الذي يتطلب استعمال تكنولوجيا ملائمتة لكل شكل من الطاقت البديلت، فالطاقت الشمسيت هي طاقت الموجات الكهرومغناطيسيت المكونت لأشعت الشمس وتتجسد على الأرض بعدة أشكال منها الضوء والحرارة، أما الطاقت الهوائيت ففي حركت الهواء نفسـه وهي بدلك طاقت ميكانيكيت. إن ضعف تركيز الطاقت ِِْْ بعض المصادر البديلت والطاقت الشمسيت بالذات يتفق مع كثافت الطاقت المطلوبت في العديد من نقاط الاستهلاك، وتتضح صحت هذه العلاقتة وتتبلور بشكل أفضل إذا مـا اتبعت الإجراءات الكفيلت بتقليل استهلاك الطاقت. 3-1 - مزايا استخدام الطاقت المتجددة وتكنولوجياتها: تتميز مصادر الطاقت المتجددة بتنوع وتعدد استخداماتها، حيث تستخدم يِّ العديد من المجالات، مثل توليد الكهرباء، والاستخدامات المنزليت الصغيرة (الطبخ والتدفئت)، والمجالات الصناعيت، وتحليه المياه. لذلك فإن استخدام مصادر الطاقت المتجددة يحقق العديد من المزايا التاليت [12]:

$$
\text { 1 } 1 \text { 3. }
$$

1_4 معوقات نشر الطاقت المتجددة: تتمثل المعوقات التي تجابه نشر الطاقت المتجدددة في الآتي [13]: معوقات فنيت: تتمثل أهمها يِّ:

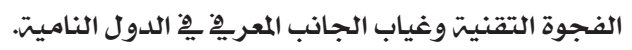
معوقات تسويقيت وغياب تعريف المستهلك بتطبيقات الطاقت المتجددة المنزليت (التسخين الشمسي للمياه، الإضـاءة...). انخفاض مستوى خدمات ما بعد البيع (التشغيل والصيانت). معوقات تشريعيت: يتمثل أهمها يِّ: فرض الضرائب الجمركيت على معدات الطاقت المتجددة وقصور التمويل المحلى. - محن. محدوديت مشاركت القطاع الخاص يِّ إنشاء مشروعات لإنتاج واستخدم تطبيقات الطاقت المتجددة. التحديات التي تواجه توظيف الطاقات المتجددة على الصعيد

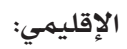
عدم وجود استراتيجيات ملائمتة وشاملتة على المستوى الحكومي أو القطاع الخاص لتمويل المشاريع المتعلقت باستخدامات الطاقات المتجددة كبديل للطاقت التقليديت التي ستتضب يوماً ما ولن تفي بمتطلبات الدول العربيت يْ المستقبل. 


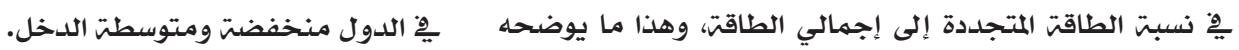
- تطورت ذسبت الطاقات المتجددة بمعدل متوسط قدر

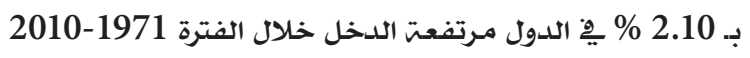

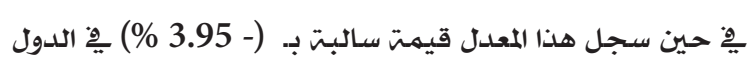
الشكل 2 والذي يمكن أن نستخلص منه النتائج التاليت:

منخفضت ومتوسطت الدخل خلال نفس الفترة. لقد تضاعفت نسبت الطاقات المتجددة ِِِ الدول المرتفعت الدخل 2.2 مرة بين سنتي 1971 و2010، يِّ حين تضاعفت 0.14 مرة

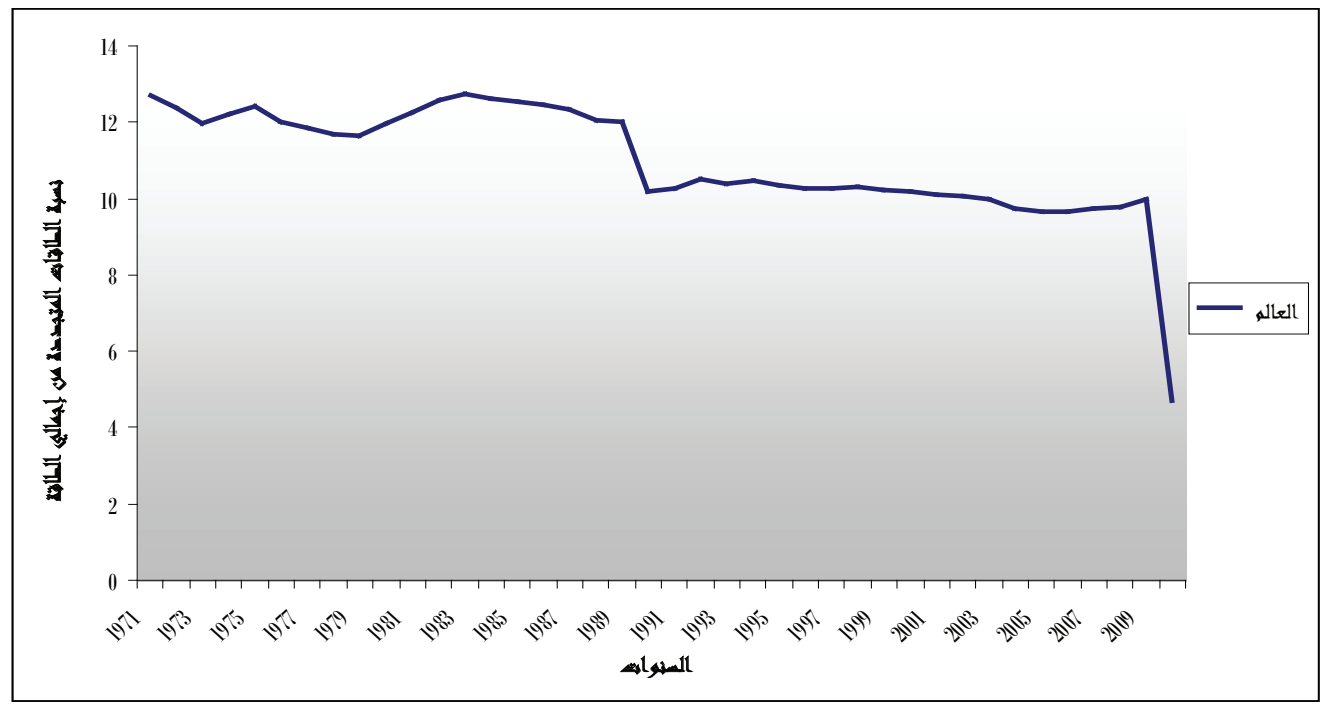

الشككل (1). نسبت الطاقت المتجددة من إجمالي الطاقتم في العالم خلال الفترة 1971-2010

المصدر: من إعداد الباحثين بالاعتماد على World Bank, Database 2011، وباستخدام برنامج Excel.

للاشتعال الكتلت الحيويت الصلبت والسـائلتة، والغاز الحيوي،

والمخلفات الصناعيت، ومخلفات البلديات، مقاست كلها

كنسبت مئويت من إجمالي استهلاك الطاقتة.

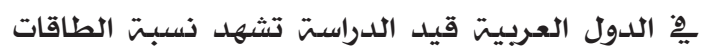
المتجددة من إجمالي استهلاك الطاقت استقرارا خلال الفترة 1995-2009 حيث هذه النسبت تبقى يِ حدود 7.5 \% إلى الى 9.5 \% حيث بلغت النسبت \% 9.43 سنت 1995 و8.93 \%

سنت 2000 وانخفضت أكثر سنت 2009 إلى 7.63 \% إذا أخذنا بعـين الاعتبار الهيكل الاقتصادي للدول العربيت قيد الدراست من خلال تقسيهها إلى دول نفطيت ودول غير نفطيت نجد أن نسبت الطاقات المتجددة من إجمالي استهلاكك الطاقتة يِّ الدول غير النفطيت أكبر من نفس المس

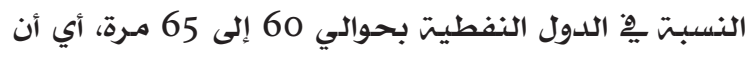
الدول النفطيت يبقى تركيزها على المحروقات كحصت المصني

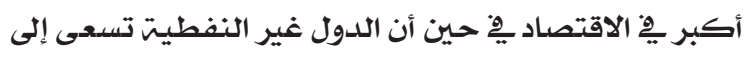
تنويع الاقتصاد أكثر والبحث عن مصدادر أخرى للنهو من خلال التوجه إلى الطاقات المتججددة وهذا مـا يوضحهـ الشكل 4.
إن نتائج البيان السـابق لا تعطينا أسبـاب واضحت عن الاتجاه السـالب لنسبتت الطاقات المتجدلدة إلى إجمالي الطاقتت، وعند تحليل جغرافيت التوجه إلى الطاقت المتجددة يتضح لنا جليـا أن الدول مرتفعت الدخل هي السباقت إلى التوجهه الى الطاقت البـديلتة، مِّ حسين أن الدول منخفضت ومتوسطت الدخل تشهد معدلات متناقصت ِِِ نسبتت الطاقت المتجددة إلى إجمالي الطاقت، وهذا مـا يوضحهـ الشكل 2 والذي يهكن أن نستخلص منـه النتائج التاليتي: لقد تضاعفت نسبت الطاقات المتجددة ٍِِ الدول المرتفعت الدخل 2.2 مـرة بين سنتي 1971 و2010، تضاعفت 0.14 مـرة ٍِِ الدول منخفضت ومتوسطت الدخل. تطورت نسبت الطاقات المتجددة بمعدل متوسط قدر بـ 1.2 عْ الدول مـرتفعت الدخل خلال الفترة 1971-2010

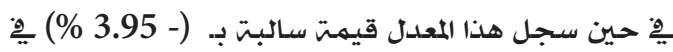
الدول منـخفضت ومتوسطت الدخل خلال نفس الفترة.

المحور الثالث: تحليل توجه الدول العربية إلى الطاقات المتجددة تشهل مصادر الطاقت المتجددة والمخلفات القابلت 


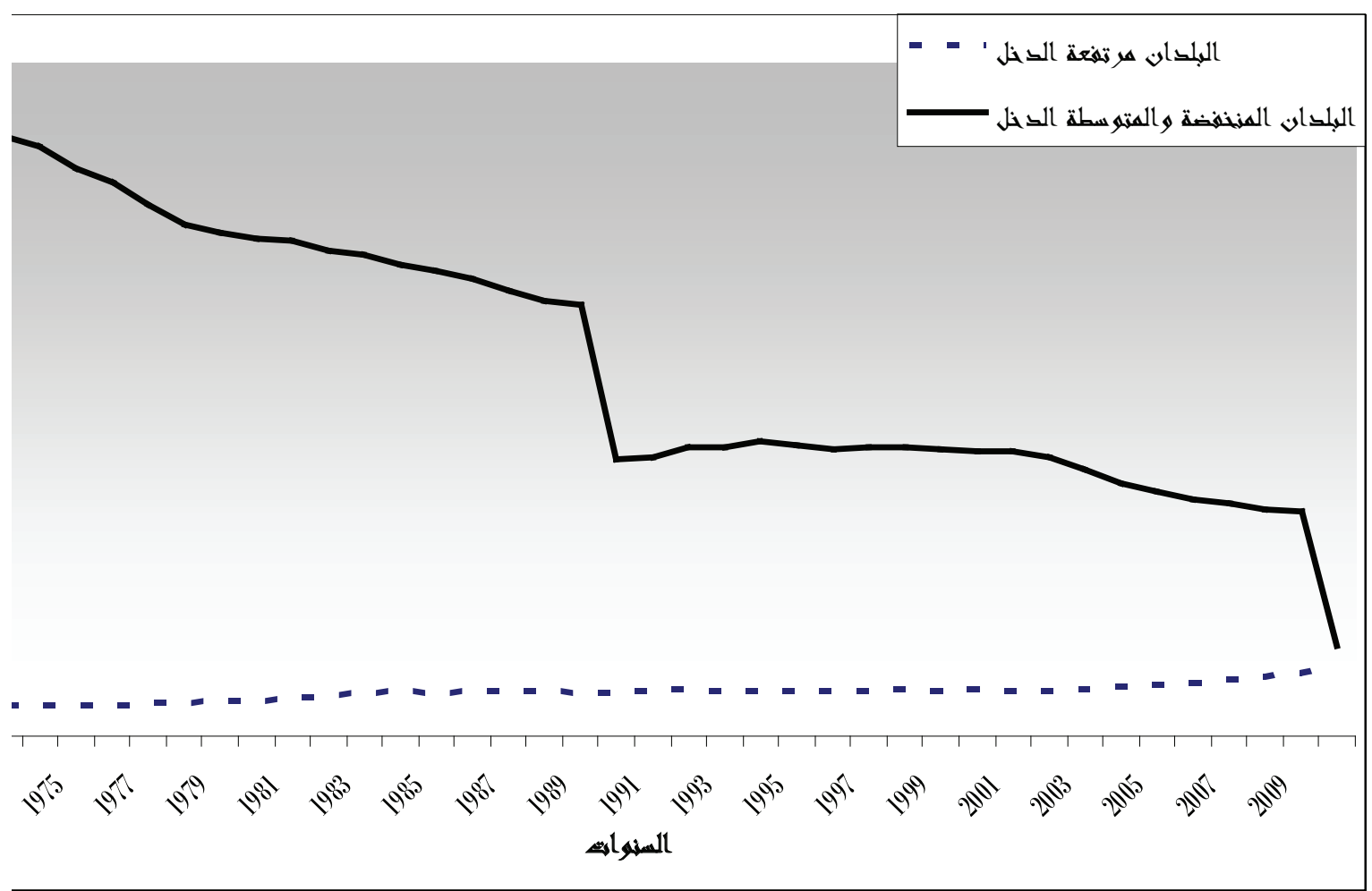

الشكل (2). تطور نسبت التوجه إلى الطاقات المتجددة في البلدان مرتفعت الدخل والبلدان منخفضت ومتوسطت الدخل خلال الفترة 1971-2010

المصدر: من إعداد الباحثين بالاعتماد على World Bank, Database 2011 :Excel وباستخدام برنامج

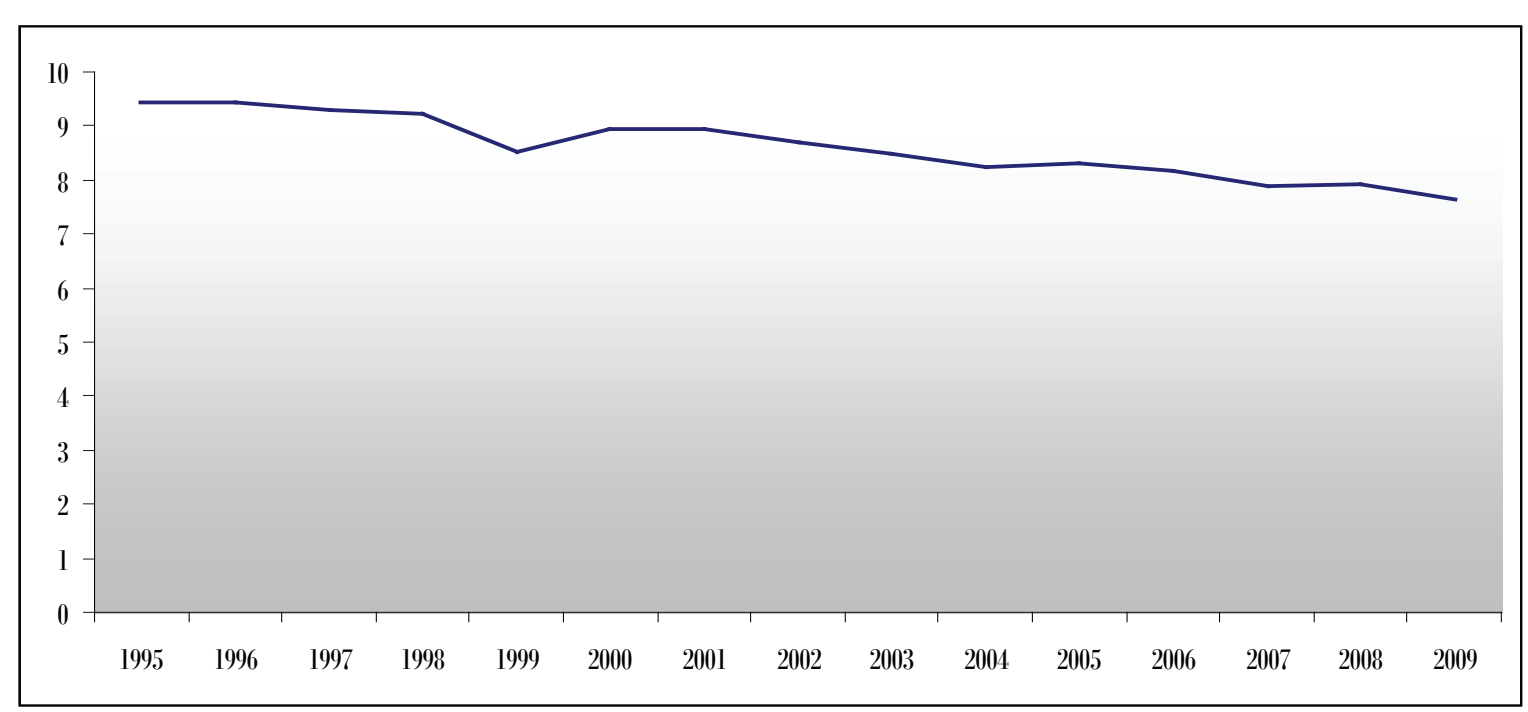

الشكل (3). نسبت الطاقات المتجددة من إجمالي استهلاك الطاقت في عينت من الدول العربيت خلال الفترة 1995-2009

المصدر: من إعداد الباحثين بالاعتماد على World Bank, Database 2011 وباستخدام برنامج Excel. 


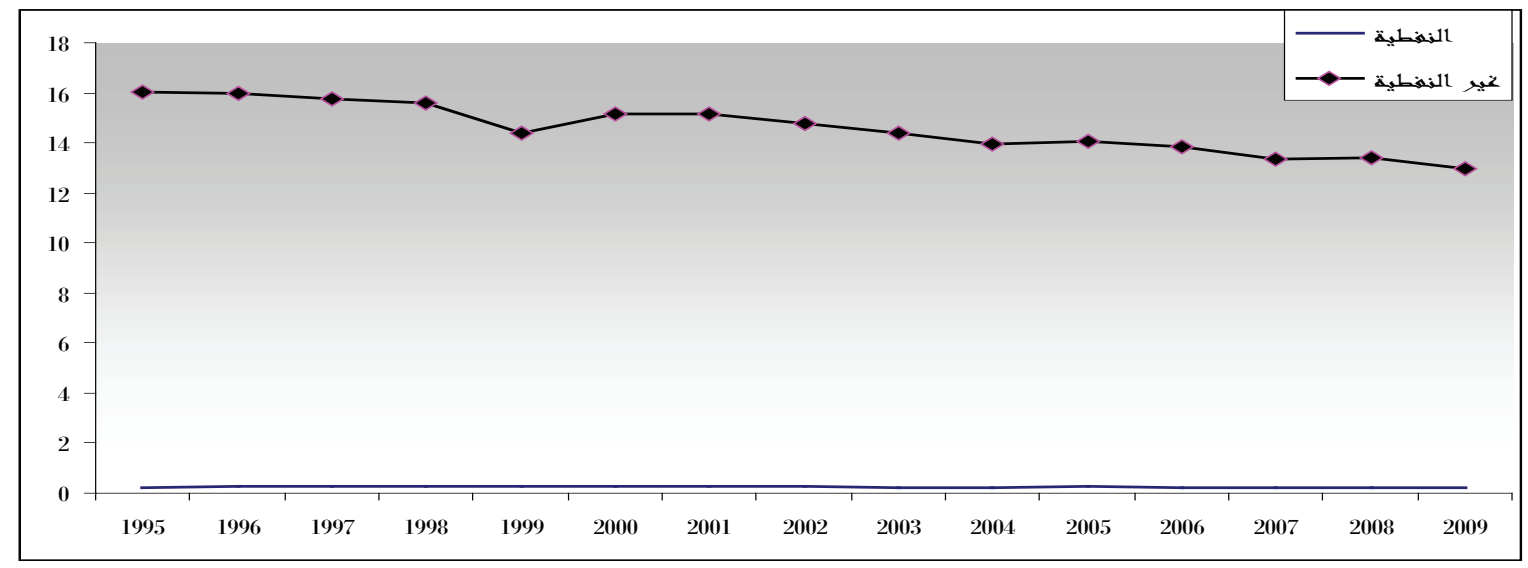

الشكل (4). نسبت الطاقات المتجددة من إجمالي استهلاك الطاقت في الدول النفطيت وغير النفطيت خلال الفترة 2009-1995

المصدر: من إعداد الباحثين بالاعتماد على World Bank, Database 2011 وباستخدام برنامج Excel.

\section{المحور الرابع: التنبؤ بتوجه الدول العربية إلى الطاقات المتجددة خلال الفترة 2016-2010}

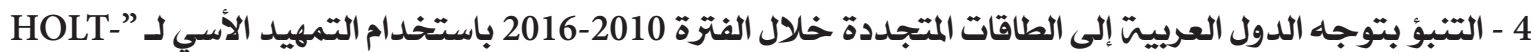
ـ WINTERS

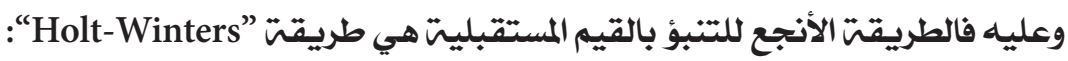

1-4 - تحديد قيم المعلمات م و م : يمكن تلخيص النتائج في الجدول التالي:[14]

الجدول (1). قيم المعلمات ه و م في طريقتت التمهيد الأسي لـ "Holt-Winters

\begin{tabular}{|c|c|c|}
\hline$\beta$ & $\alpha$ & $-\pi$ \\
\hline 0.17 & 0.84 & الجزائر \\
\hline 1.00 & 0.13 & مصر \\
\hline 1.00 & 0.70 & العراق \\
\hline 0.07 & 1.00 & السعوديت \\
\hline 0.00 & 1.00 & لينان \\
\hline 0.00 & 0.94 & Jييـيا \\
\hline 0.01 & 0.64 & المخرب \\
\hline 1.00 & $\begin{array}{l}0.11 \\
\end{array}$ & السودوان \\
\hline 0.00 & 1.00 & سوريا \\
\hline 0.32 & 0.53 & تونس \\
\hline 0.14 & 0.90 & الإمارات \\
\hline 0.24 & 1.00 & اليهـن \\
\hline
\end{tabular}

المصدر: من إعداد الباحثين بالاعتماد على مخرجات برنامج Eviews7.

من خلال نتائج التنبؤ يلاحظ أن هناك نتائج متباينت

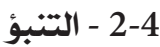

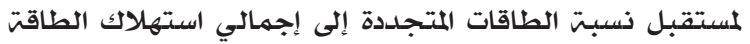

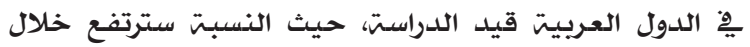
بعد تعويض قيم المعلمات المتحصل عليها بِّْ النموذج لكل سلسلت حصلنا على القيم التنبؤيت، حيث توضح السلسلتة

الفترة 2010-2016 يِّ المجموعت التي تضهم ككلا من العراق، ليبيا، تونس، وستكون معدلات نمو النسبت ِِّ العراق 3.06 \%

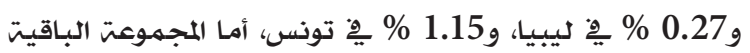

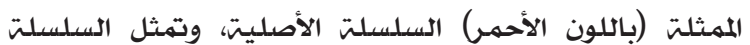

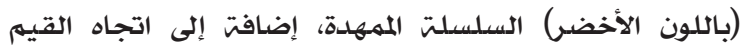
التنبؤيت [15]. 
انخفاض النسبت المعنيت. إضافت إلى ذلك يلاحظ أن نسبت الدول

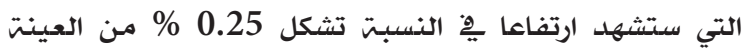

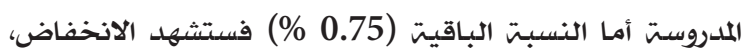
فالدول التي تشهد الارتفاع ِِّ النسبتة تمثلها 66.67 \%

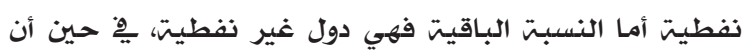
الدول التي تشهد انخفاض النسبت تمثل 66.67 \% نفطيت أما النسبت الباقيت فهي دول نفطيت.
فستتجه نسبت الطاقات المتجددة إلى إجمالي استهلاك الطاقت إلى الانخفاض وستكون نسب الانخفاض: - 14.56 \% 14.69 \%

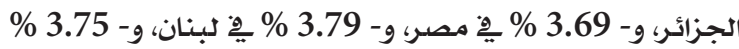

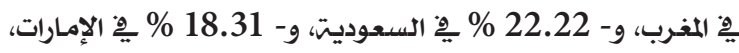

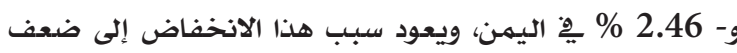

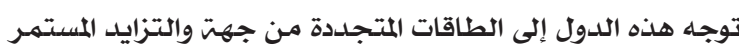
هِِ استهلاك الطاقت بأنواعها من جهت أخرى وهذا مـا يؤدي إلى لى

الجدول (2). نتائج التتبؤ بنسبت الطاقات المتجددة إلى إجمالي استهلاكك الطاقت باستخدام طريقتمالتمهيد الأسي لـ

\begin{tabular}{|r|r|r|r|r|r|r|r|r|r|r|r|r|}
\hline $\begin{array}{c}\text { Country } \\
\text { Name }\end{array}$ & Algeria & Egypt & Iraq & Lebanon & Libya & Morocco & $\begin{array}{r}\text { S a u d i } \\
\text { Arabia }\end{array}$ & Sudan & Syria & Tunisia & $\begin{array}{c}\text { United } \\
\text { Arab } \\
\text { Emmirates }\end{array}$ & $\begin{array}{c}\text { Yemen } \\
2010\end{array}$ \\
\hline 0,12813 & 1,97105 & 0,0831 & 1,76993 & 0,82775 & 3,04337 & 0,00233 & 66,86607 & 0,02778 & 14,12345 & 0,02516 & 1,29729 \\
\hline 2011 & 0,11504 & 1,90476 & 0,08585 & 1,70884 & 0,83001 & 2,93929 & 0,00183 & 64,99741 & 0,02701 & 14,29017 & 0,02219 & 1,26731 \\
\hline 2012 & 0,10195 & 1,83846 & 0,08859 & 1,64775 & 0,83226 & 2,8352 & 0,00132 & 63,12876 & 0,02624 & 14,45689 & 0,01923 & 1,23734 \\
\hline 2013 & 0,08885 & 1,77217 & 0,09133 & 1,58666 & 0,83452 & 2,73112 & 0,00081 & 61,2601 & 0,02547 & 14,62361 & 0,01626 & 1,20736 \\
\hline 2014 & 0,07576 & 1,70588 & 0,09407 & 1,52557 & 0,83678 & 2,62703 & 0,0003 & 59,39145 & 0,0247 & 14,79033 & 0,01329 & 1,17739 \\
\hline 2015 & 0,06267 & 1,63959 & 0,09681 & 1,46448 & 0,83904 & 2,52294 & 0,00021 & 57,5228 & 0,02393 & 14,95705 & 0,01032 & 1,14741 \\
\hline 2016 & 0,04957 & 1,57329 & 0,09955 & 1,40339 & 0,8413 & 2,41886 & 0,00031 & 55,65414 & 0,02317 & 15,12377 & 0,00736 & 1,11744 \\
\hline
\end{tabular}

المصدر: من إعداد الباحثين بالاعتماد على مخرجات برنامج SPSS10.

5

من خلال النتائج المتوصل إليها مـن الاختبارات تم التوصل إلى أن سلسلت المستوى، والجدول 3 يوضح هذه النتائج.

$$
\text { 5-2 تقدير النموذج الإجمالي: }
$$

من خلال دراست الاستقراريت للسلسلتين يمكن تقدير النهموذج التالي:

$$
T C R_{i t}=b_{0}+b_{1} \cdot E N R G_{i}+\varepsilon_{i}
$$

إن عمليت التقدير أعطت النتائج التاليت:
المحور الخامس: أثر التوجه إلى الطاقات المتجددة على

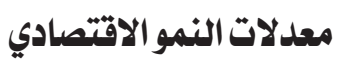

5 - أثر التوجه إلى الطاقات المتجددة على معدلات النمو

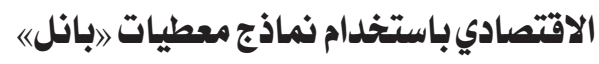

يتكون النموذج من متغير نسبتة الطاقات المتجددة من إجمالي الطاقت كمتغير مستقل ENRG أكسيد الكريون كمتغير تابع TCR 2009-1995 لعدد 12 دولت عربيتة، وسنقوم ِِّ بدايت الدراستة بدراست استقراريت السلاسل الزمنيت لهذين المتغيرين.

$$
\begin{aligned}
& T C R_{t}=\underset{\substack{8.8581 \\
(0.0000)}}{0.1048}-\underset{\substack{0.0104 \\
(0.9917)}}{5.39 \times 10^{-6}} \cdot E N R G t+\varepsilon_{i t} \\
& R^{2}=0.000001 \ldots . D W=1.63 \ldots . F=0.0001(0.9916)
\end{aligned}
$$

الاقتصاديت، بحيث أن ارتفاع نسبت الطاقات المتجددة بـ 1 \% تسمـح بتخفيض معدلات النمو الاقتصدادي ب. 0.00000539 \%؛ وهذا

التأثير السلبي يقدر بـ 0.0001 \% وهي نسبت ضعيف ضعيفت جدا.

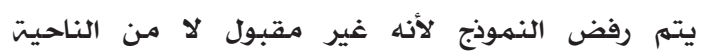
الاقتصاديت من خلال التأثير السلبي لنسبت الطاقات المتجددة

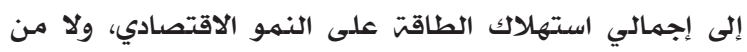

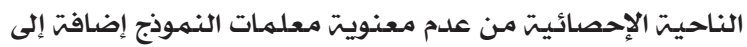
مشكلت الارتباط الذاتي لكلأخطاء.
معلمات النموذج ذات معنويت إحصائيت مـا عدا المعلمت من خلال اختبار فيشر يتضـح عدم المعنويت الكليت لمعلمات النهموذج.

إحصائيت "دربين-واتسون) تثبت عدم وجود ارتباط ذاتي كلأخطاء. هناك علاقت عكسيت بين التوجه إلى الطاقات المتجددة ومعدلات النمو الاقتصادي وهذا غير مقبول من الناحيت 
الجدول (3). نتائج اختبارات الاستقراريت عند المستوى.

\begin{tabular}{|c|c|c|c|c|c|c|c|c|c|c|c|}
\hline & \multicolumn{3}{|c|}{ النموذج 1} & \multicolumn{3}{|c|}{ النموذج 2} & \multicolumn{3}{|c|}{ النموذج 3} & \multirow[b]{2}{*}{ الاختبار } & \multirow[b]{2}{*}{ المتغير } \\
\hline 牙 & 傁 & 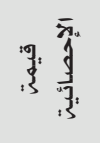 & $\frac{\hat{3}}{3}$ & 空 & is & 余 & 高 & "g. & $\begin{array}{l}\frac{3}{3} \\
\frac{3}{3} \\
\frac{3}{3}\end{array}$ & & \\
\hline \multirow{4}{*}{ 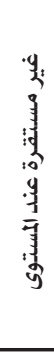 } & 0.0000 & 4.87- & \multirow{4}{*}{2} & 0.0070 & 2.45- & \multirow{4}{*}{2} & 0.0000 & 24.43- & \multirow{4}{*}{2} & $\begin{array}{l}\text { Levin, Lin \& } \\
\text { Chu } t^{*}\end{array}$ & \multirow{4}{*}{$E N R G_{\dot{i t}}$} \\
\hline & & & & & & & 0.8227 & 0.92 & & Breitung t-stat & \\
\hline & 0.0000 & 81.26 & & 0.0046 & 40.56 & & 0.0000 & 62.85 & & $\begin{array}{l}\text { ADF-Fisher } \\
\text { Chi-square }\end{array}$ & \\
\hline & 0.0000 & 112.73 & & 0.0022 & 48.49 & & 0.0000 & 69.91 & & $\begin{array}{l}P P \text { - Fisher Chi- } \\
\text { square }\end{array}$ & \\
\hline \multirow{4}{*}{ 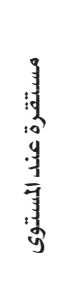 } & 0.0000 & 5.95- & \multirow{4}{*}{2} & 0.0000 & 5.69- & \multirow{4}{*}{1} & 0.0000 & 4.50- & \multirow{4}{*}{1} & $\begin{array}{c}\text { Levin, Lin \& } \\
\text { Chu } t^{*}\end{array}$ & \multirow{4}{*}{$T C R_{i t}$} \\
\hline & & & & & & & 0.9807 & 2.06 & & Breitung t-stat & \\
\hline & 0.0000 & 71.28 & & 0.0000 & 67.11 & & 0.0039 & 46.47 & & $\begin{array}{l}\text { ADF - Fisher } \\
\text { Chi-square }\end{array}$ & \\
\hline & 0.0000 & 74.52 & & 0.0000 & 66.06 & & 0.0105 & 42.80 & & $\begin{array}{l}P P \text { - Fisher Chi- } \\
\text { square }\end{array}$ & \\
\hline
\end{tabular}

المصدر: من إعداد الباحثين بالاعتماد على مخرجات برنامج Eviews7.

$$
\text { ونتائج تقدير النماذج موضحت فيما يلي: }
$$$$
5 \text { - } 3 \text { - تقدير النماذج ذات الأثر الثابت }
$$

يتم تقدير هذه النماذج من خلال تقدير نموذج الأثر 1-3-5 - تقدير نموذج الأثر الثابت للأفراد (الدول): من خلال تقدير النموذج حصلنا على النتائج التاليت:

$$
\begin{aligned}
& \left.T C R_{i t}=\begin{array}{r}
0.2057 \\
3.7237 \\
(0.0003)
\end{array} \quad \begin{array}{r}
0.1 .0117 \\
(0.0639
\end{array}\right) \\
& R^{2}=0.0595 G_{i t}+\varepsilon_{i t}
\end{aligned}
$$

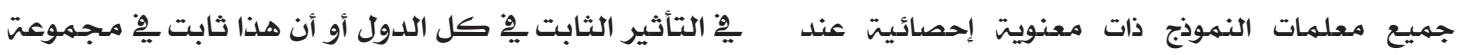
الدول المدروست وتعطى صيغتت الإحصائيت كما يلي:

$$
F=\frac{\left(R_{1}^{2}-R_{0}^{2}\right)}{\left(1-R_{1}^{2}\right) /(n T-n-K)} \rightarrow F(n-1, n T-n-K)
$$

حيث: بينما K تمثل عدد المتغيرات المفسرة.

إذا كان $H_{0}$ نفرض الفرضيت

$$
\text { (عدم وجود أثر فردي). }
$$

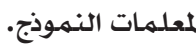

إحصائيت ״دربين-واتسونه تثبت عدم وجود ارتباط ذاتي

كلأخطاء.

من خلال النموذج يلاحظ أنه كلما ارتفعت نسبت الطاقات المتجددة انخفضت معدلات النمو الاقتصادي،

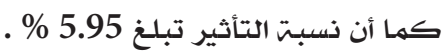
2-3-5 - اختبار وجود الأثر الثابت الفردي يسمح اختبار فيشر بمعرفت ما إذا كان هناك اختلاف 
الجدول (4). اختبار وجود أو عدم وجود أثر ثابت للأفراد (الدول).

\begin{tabular}{|c|c|c|c|}
\hline \multicolumn{4}{|c|}{$\begin{array}{l}\text { Redundant Fixed Effects Tests } \\
\text { Equation Untitled } \\
\text { Test cross-section fixed effects }\end{array}$} \\
\hline Effects Test & Statistic & $\mathrm{df}$ & Prob \\
\hline $\begin{array}{l}\text { Cross-section F } \\
\text { Cross-section Chi-square }\end{array}$ & $\begin{array}{c}0.962011 \\
11.059076\end{array}$ & $\begin{array}{c}(11.167) \\
11\end{array}$ & $\begin{array}{l}0.4831 \\
0.4383\end{array}$ \\
\hline
\end{tabular}

المصدر: من إعداد الباحثين بالاعتماد على مخرجات برنامج Eviews7.

$$
\text { bعلمت المعت }
$$

من خلال اختبار فيشر تتضح المعنويت الكليت لمعلمات

$$
\text { النموذج. }
$$

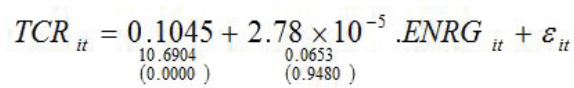$$
R^{2}=0.3709 \ldots . D W=1.62 \ldots . . F=6.4466(0.0000)
$$

إحصائيت "دريين-واتسون" تثبت عدم وجود ارتباط ذاتي لكلأخطاء.

من خلال النموذج يلاحظ أنه كلما ارتفعت نسبت الطاقات المتجددة ارتفعت معدلات النمو الاقتصادي، كما أن نسبت التأثير تبلـ 37.09 \%

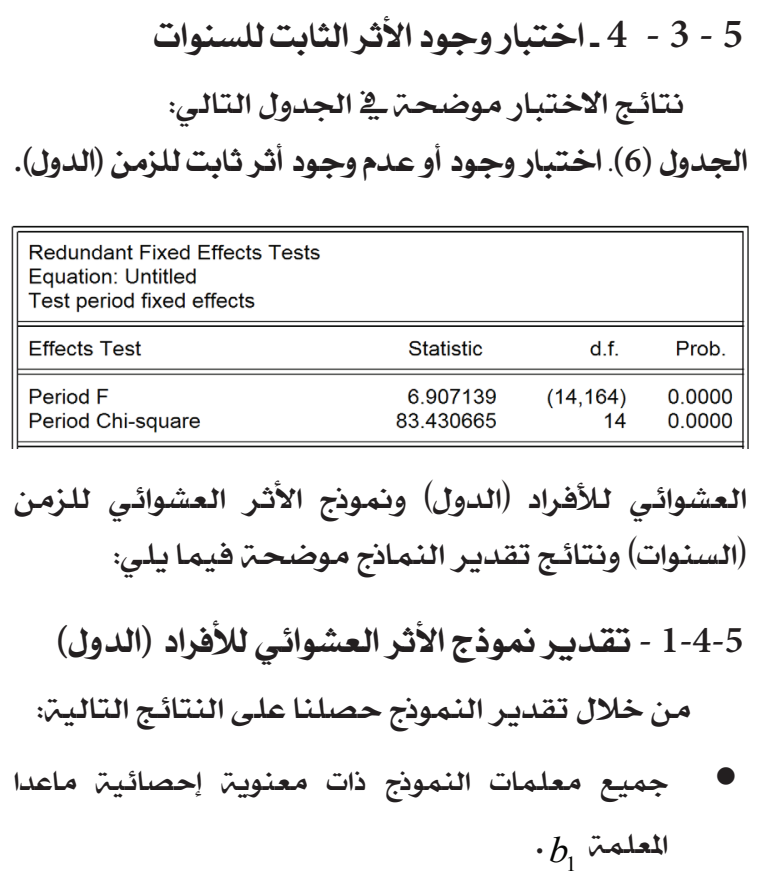

$$
\text { المعلمت }
$$

من خلال اختبار فيشر تتضح عدم المعنويت الكليت
بما أن الاحتمال يسـاوي (0.4831) وهو أكبر من 5 \% فهذا يعني أن $\quad F \prec F_{0.05}^{n-1, n T-n-K}$

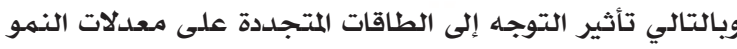

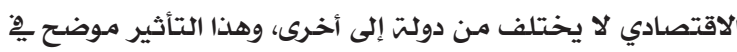

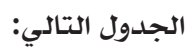
الجدول (5). أثر الأفراد (الدول)

\begin{tabular}{|l|c|}
\hline \multicolumn{1}{|c|}{ Country } & Effect \\
\hline Algeria & -0.113709 \\
\hline Egypt & -0.079523 \\
\hline Iraq & -0.041156 \\
\hline Lebanon & -0.083810 \\
\hline Libya & -0.117127 \\
\hline Morocco & -0.079480 \\
\hline Saudi Arabia & -0.128401 \\
\hline Sudan & 0.828045 \\
\hline Syria & -0.085858 \\
\hline Tunisia & 0.014672 \\
\hline United Arab Emirates & -0.071335 \\
\hline Yemen & -0.042318 \\
\hline
\end{tabular}

المصدر: من إعداد الباحثين بالاعتماد على مخرجات برنامج -0views7. 5 - 3 - 3 - تقدير نموذج الأثر الثابت للزمن (السنوات) من خلال تقدير النموذج حصلنـا على النتائج التاليت: جميع معلمات النهوذج ذات معنويت إحصائيت ماعدا المصدر: من إعداد الباحثين بالاعتماد على مخرجات برنامج Eviews7. بما أن الاحتمال يساوي (0.0000) وهو أقل من 0.05 فإن هناك غياب أثر ثابت للسنوات وبالتالي تأثير التوجه إلى الطاقات المتجددة على معدلات النمو الاقتصادي يختلف من سنت إلى أخرى، وهذا التأثير موضـح ِِّ الجدول 7.

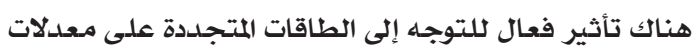
النهو الاقتصادي ِِّ الفترة الأخيرة (2003-2008) إضافت إلى إلى سنتي 1999 و2000 ِِّ حين أن هذا التأثير غير فعال ِِّ الفترات

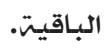
5-4 تقدير النماذج ذات الأثر العشوائي

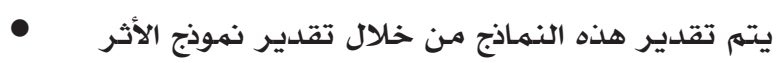


الطاقات المتجددة انخفضت معدلات النمو الاقتصادي،

كلأخطاء.

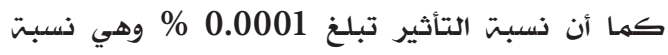

ضعيفت جدا إحصائيا.

من خلال النموذج يلاحظ أنه كلما ارتفعت نسبت

الجدول (7). أثر الزمن (السنوات)

\begin{tabular}{|c|c|c|c|c|c|c|c|c|c|c|c|c|c|c|c|}
\hline DATEID & 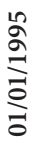 & 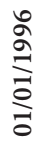 & $\begin{array}{l}\hat{\sigma} \\
\stackrel{a}{\partial} \\
\text { aे }\end{array}$ & $\begin{array}{l}\stackrel{\infty}{2} \\
\stackrel{2}{\Xi} \\
\stackrel{0}{0}\end{array}$ & $\begin{array}{l}\stackrel{\partial}{\partial} \frac{1}{\partial} \\
\text { aे }\end{array}$ & 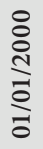 & $\begin{array}{l}\overline{\grave{O}} \\
\stackrel{0}{0} \\
\text { a }\end{array}$ & 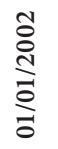 & 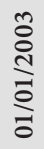 & 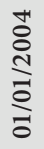 & 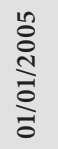 & 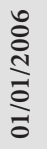 & 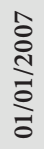 & 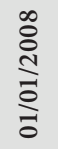 & $\begin{array}{l}\stackrel{\partial}{0} \\
\stackrel{0}{0} \\
\text { a }\end{array}$ \\
\hline Effect & 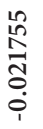 & 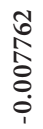 & 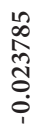 & 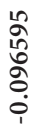 & $\begin{array}{l}\text { f } \\
\text { S } \\
0 \\
0 \\
0\end{array}$ & 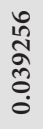 & 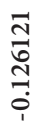 & 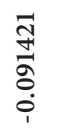 & $\begin{array}{l}\text { ma } \\
\text { o. } \\
0 \\
0 \\
0\end{array}$ & $\begin{array}{l}0 \\
\stackrel{0}{0} \\
: \\
\stackrel{0}{0}\end{array}$ & $\begin{array}{l}n \\
\hat{L} \\
0 \\
0 \\
0 \\
0\end{array}$ & $\begin{array}{l}\text { के } \\
0 \\
\hat{\infty} \\
\infty \\
0 \\
0\end{array}$ & 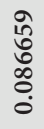 & $\begin{array}{l}\text { No } \\
\text { Oे } \\
\stackrel{0}{0}\end{array}$ & ర్ \\
\hline
\end{tabular}

المصدر: من إعداد الباحثين بالاعتماد على مخرجات برنامج Eviews7.

$$
\text { المعلمت }
$$

الجدول (9). أثر الأفراد (الدول)

\begin{tabular}{|l|r|}
\hline Country & Effect \\
\hline Algeria & 0.000000 \\
\hline Egypt & 0.000000 \\
\hline Iraq & 0.000000 \\
\hline Lebanon & 0.000000 \\
\hline Libya & 0.000000 \\
\hline Morocco & 0.000000 \\
\hline SaudiArabia & 0.000000 \\
\hline Sudan & 0.000000 \\
\hline Syria & 0.000000 \\
\hline Tunisia & 0.000000 \\
\hline United Arab Emirates & 0.000000 \\
\hline Yemen & 0.000000 \\
\hline
\end{tabular}

المصدر: من إعداد البـاحثين بالاعتماد على مخرجات برنامج Eviews7.

من خلال اختبار فيشر تتضح عدم المعنويت الكليت لمعلمات النموذج. إحصائيت "دربين-واتسونه تثبت عدم وجود ارتباط ذاتي كلأخطاء.

من خلال النموذج يلاحظ أنه كلما ارتفعت نسبت الطاقات المتجددة ارتفعت معدلات النمو الاقتصادي، كما أن نسبت التأثير تبلغ 0.0016\% وهي نسبت ضعيفت
2-4-5 - اختبار وجود الأثر العشوائي الفردي. يسهـح اختبار "هوسـمان) بهعرفتي ما إذا كان هناك اختالاف ِِّ التأثير العشوائي يِّ كل الدول أو أن هذا عشوائي

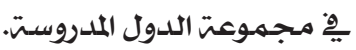

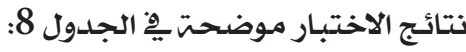

الجدول (8). اختبار وجود أو عدم وجود أثر عشوائي لنأفراد (الدول).

\begin{tabular}{|c|c|c|c|}
\hline \multicolumn{4}{|c|}{$\begin{array}{l}\text { Correlated Random Effects - Hausman Test } \\
\text { Equation: Untitled } \\
\text { Test cross-section random effects }\end{array}$} \\
\hline Test Summary & Chi-Sq. Statistic & Chi-Sq. d.f. & Prob. \\
\hline Cross-section random & 3.499836 & 1 & 0.0614 \\
\hline
\end{tabular}

المصدر: من إعداد الباحثين بالاعتماد على مخرجات برنامـج

.Eviews7

بما أن الاحتمال يساوي (0.0614) وهو أكبر من 5 \% فإن هناك ارتباطا للآثار الفرديت والمتغيرة المفسرة أي أن أثر أثر الدول غير عشوائي وبالتالي لا يوجد تأثير، غير أن هذه القوة

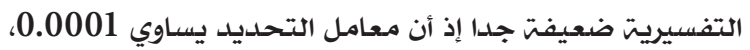
والجدول 9 يوضـح عدم وجود أي تأثير.

3-4-5 - تقدير نموذج الأثر العشوائي للزمن (السنوات) من خلال تقدير النموذج حصلنا على النتائج التاليت:

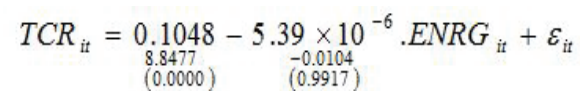
$R^{2}=0.000001 \ldots . D W=1.63 \ldots . . F=0.0001(0.9916)$

جميع معلمات النموذج ذات معنويتت إحصائيت ماعدا 


$$
\begin{aligned}
& 5 \text { - } 4 \text { - } 4 \text { - اختبار وجود الأثر العشوائي للسنوات } \\
& \text { نتائج الاختبار موضحت يِّ الجدول 10: } \\
& \text { الجدول (10). اختبار وجود أو عدم وجود أثر عشوائي للزمن (الدول) }
\end{aligned}
$$

\begin{tabular}{||lrrr||}
\hline $\begin{array}{l}\text { Correlated Random Effects - Hausman Test } \\
\text { Equation: Untitled } \\
\text { Test period random effects }\end{array}$ & & & \\
\hline \hline Test Summary & Chi-Sq. Statistic & Chi-Sq. d.f. & Prob. \\
\hline \hline Period random & 1.265762 & 1 & 0.2606 \\
\hline \hline
\end{tabular}

المصدر: من إعداد الباحثين بالاعتماد على مخرجات برنامج Eviews7.

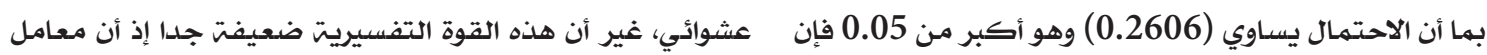

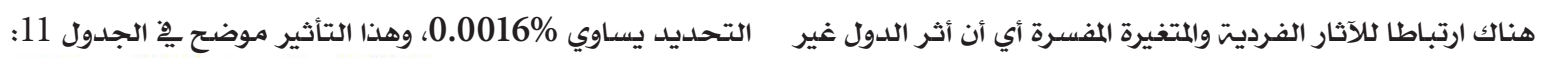
$T C R_{\text {it }}=\underset{\substack{4.3781 \\(0.0000)}}{0.1045}+\underset{\substack{0.0538 \\(0.9571)}}{2.29 \times 10^{-5}} \cdot E N R G_{\text {it }}+\varepsilon_{\text {it }}$ $R^{2}=0.000016 \ldots . . D W=1.62 \ldots . . F=0.00289(0.9571)$

\begin{tabular}{|c|c|c|c|c|c|c|c|c|c|c|c|c|c|c|c|}
\hline DATE & $\frac{\sqrt[n]{\sigma}}{\stackrel{\sigma}{\sigma}}$ & 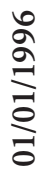 & 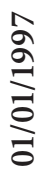 & 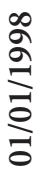 & 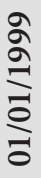 & 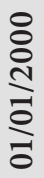 & 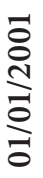 & 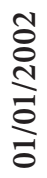 & 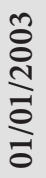 & 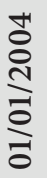 & 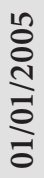 & $\begin{array}{l}\text { ○ } \\
8 \\
\text { ㄱ } \\
\text { - } \\
0 \\
0\end{array}$ & 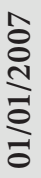 & 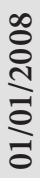 & $\begin{array}{l}\stackrel{a}{\delta} \\
\stackrel{-}{0} \\
0 \\
0 \\
0\end{array}$ \\
\hline Effect & $\begin{array}{l}\text { भै } \\
1 \\
\infty \\
0 \\
0 \\
0 \\
\end{array}$ & 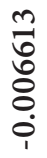 & 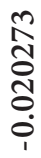 & $\begin{array}{l}\text { m } \\
\text { ND } \\
\infty \\
0 \\
0 \\
0\end{array}$ & $\begin{array}{l}10 \\
\text { ని } \\
\infty \\
0 \\
0 \\
0\end{array}$ & 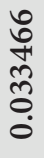 & $\begin{array}{l}\frac{J}{n} \\
\hat{n} \\
0 \\
\stackrel{0}{0}\end{array}$ & $\begin{array}{l}\text { ñ } \\
\text { م̂} \\
\text { No } \\
0 \\
0\end{array}$ & 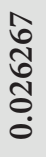 & $\begin{array}{l}\stackrel{0}{\pi} \\
\text { రె} \\
\stackrel{2}{0} \\
\stackrel{0}{0}\end{array}$ & 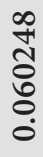 & 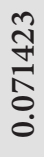 & $\begin{array}{l}\text { N } \\
\infty \\
\\
0 \\
0\end{array}$ & 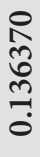 & $\begin{array}{l}\vec{F} \\
\text { N } \\
\text { in } \\
\dot{0}\end{array}$ \\
\hline
\end{tabular}

الجدول (11). أثر الزمن (السنوات)

المصدر: من إعداد الباحثين بالاعتماد على مخرجات برنامج Eviews7.

المتجددة من إجمالي استهلاك الطاقت استقرارا خلال

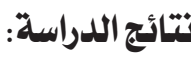
الفترة 1995-2009 حيث هذه النسبت تبقى ِِ حدود \% 9.5 \% 7.5 نتائج التنبؤ تبين أن هناك نتائج متباينت لمستقبل نسبتة الطاقات المتجددة إلى إجمالي استهلاك الطاقت يِّ الدول العربيتة قيد الدراستة، حيث النسبتة سترتفع خلال الفترة 2016-2010 ِِّْ المجموعت التي تضمى كلا من العراق، وليبيا، وتونس، وستكون معدلات نمو النسبت فِّ العراق

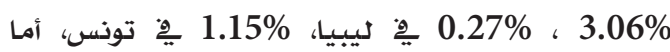
المجموعت الباقيت فستتجه نسبت الطاقات المتجددة إلى إجمالي استهلاك الطاقت إلى الانخفاض وستكون نسب

$$
\begin{aligned}
& \text { معدل نهو حصت الطاقات المتجددة على المستوى الدولي } \\
& \text { بلغ (- 192.36 1971) خلال الفترة 1910) حيث } \\
& \text { هذه الحصت تشكل } 12.68 \text { \% سنت } 1971 \text { ـِ حين } \\
& \text { أصبحت تمثل نسبت } 4.70 \text { \% سنت } 2010 . \\
& \text { تطورت نسبت الطاقات المتجددة بمعدل متوسط قدر بـ } \\
& \text { 2.10 \% يِْ الدول مـرتفعت الدخل خلال الفترة 1971-2010 }
\end{aligned}
$$

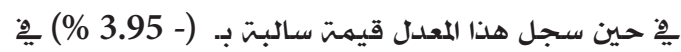

$$
\begin{aligned}
& \text { الدول منخفضت ومتوسطت الدخل خلال نفس الفترة. } \\
& \text { هٍِ الدول العربيت قيد الدراستة تشهد نسبت الطاقات }
\end{aligned}
$$


[5] - معهد الأبحاث التطبيقيت- القدس (أريج): (امشروع الإنارة

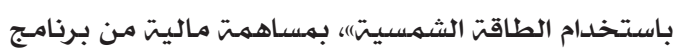

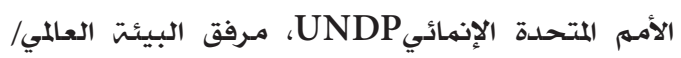
مشروع المنح الصغيرة، مؤسست هينرش بل الألمانيتضوالوكالت الاني السويسريت للتنميت والتعاون، سنت 2010، ص:03. [6] - هاني عبيد: نفس المرجع ، ص: 220. [7] - هاني عبيد: مرجع سابق ، ص: 219.

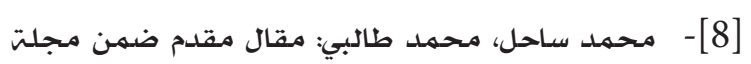

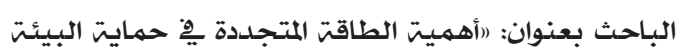
من أجل التتميت المستدامتة - عرض تجربت ألمانيا -مجلتص

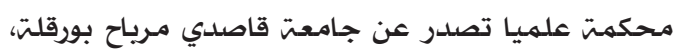

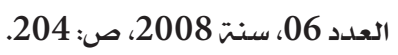

[9]- مايكل إكهارت: الطاقات المتجددة: التطلع نحو طاقت لا لا

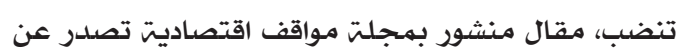

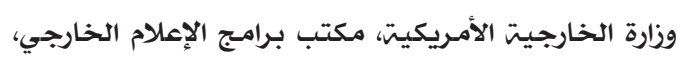
جويليت 2006، المجلد 11، العدد الثاني. [10]- Mc Mullan.J,T, Morgan, R.Murray, R.B.Energy Resource and supply John Wiley and Sons. London Energy 1976;pp:6693-.

[11]- سعود يوسف عياش: (تكنولوجيا الطاقت المتجددة)، عالم المعرفت، سلسلتة كتب ثقافيت شهريت يصدرها المجلس الوطني للثقافت والفنون والآداب، الكويت، صدرت السلسلتي

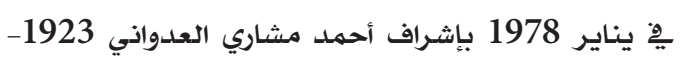
1990، سنت 1981، ص ص: 275-280.

[12]- الأمم المتحدة: (اتنميتة استخدامات الطاقت الجديدة

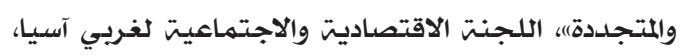

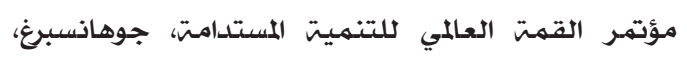

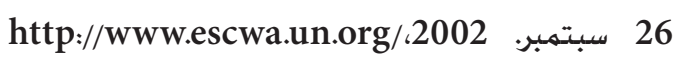
arabic/information/meetings/events/ /wssd/pdf

[13] - محمد مصطفي الخياط: (مشروع الإستراتيجيت العربيت

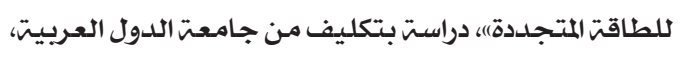
ماي 2009.

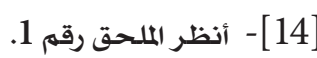
[15] - أنظر الملحق رقم 2.
الانخفاض: 14.56\%- فِ الجزائر، 3.69\%- هُ مصر،

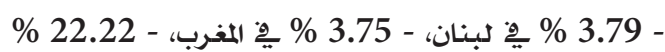
20. اليمن، من خلال تقدير نموذج الأثر الثابت للأفراد يلاحظ وجود أثر فردي ثابت وبالتالي تأثير التوجه إلى الطاقات

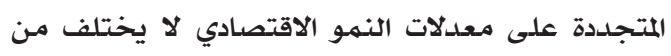
دولتة إلى أخرى. من خلال تقدير نموذج الأثر الثابت للسنوات يلاحظ عدم وجود أثر ثابت للسنوات وبالتالي تأثير التوجه إلى ندوني

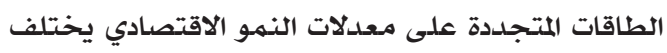
من سنت إلى أخرى.

من خلال تقدير نموذج الأثر العشوائي للأفراد يلاحظ أن

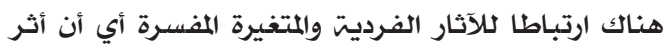

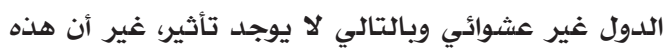
القوة التفسيريت ضعيفتً جدا إذ أن معامل التحديد يساوي 0.0001. من خلال تقدير نموذج الأثر العشوائي للسنوات أن هناك ارتباطا للآثار الفرديت والمتغيرة المفسرة أي أن أثر الدول

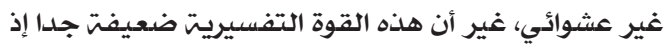
أن معامل التحديد يساوي 0.0016 \%

[1] - زرزور إبراهيه: (المسألت البيئيت والتنميت المستدامتّ، الملتقى

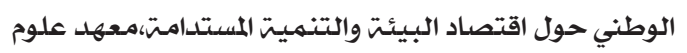
التسيير، المركز الجامعي بالمديت 2006 7-17، ص: 06 الوطنئ [2] - هاني عبيد: "لإنسان والبيئت:منظومات الطاقت والبيئت والسكان، دار الشروق، عمان ، سنترانت 2000 ، ص: 205. [3]- - محمد مصطفي الخياط، إيناس محمد إبراهيم الشيتي:

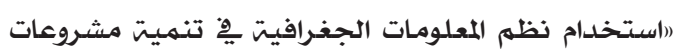

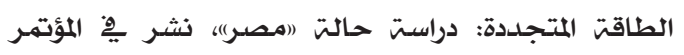
العلمي السابع عشر لنظم المعلومات وتكنولوجيا الحاسبات، القاهرة، مصر، فبراير 2010، ص: 04. [4] - منظمت التعاون الاقتصادي والتنميته، ("وكالت الطاقت):)؛

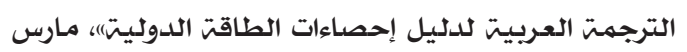
9200، ص: 121. 


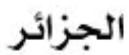

Date: 09/15/12 Time: 21:36

Sample: 19952009

Included observations: 15

Method: Holt-Winters No Seasonal

Original Series: ENRGEGY

Forecast Series: ENRGEGSM

\begin{tabular}{|llr|}
\hline \hline Parameters: & Alpha & \\
Beta & 0.1300 \\
Sum of Squared Residuals & 1.0000 \\
Root Mean Squared Error & 0.080059 \\
\hline \hline \multirow{2}{*}{ End of Period Levels: } & 0.073057 \\
& Mean & 2.064399 \\
& Trend & -0.065187 \\
\hline & & \\
\hline & & \\
\hline
\end{tabular}

Date: 09/15/12 Time: $21: 37$

Sample: 19952009

Included observations: 15

Method: Holt-Winters No Seasonal

Original Series: ENRGKSA

Forecast Series: ENRGKSSM

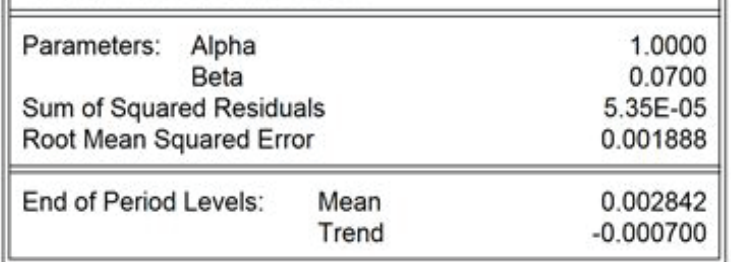

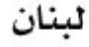

Date: 09/15/12 Time: $21: 38$

Sample: 19952009

Included observations: 15

Method: Holt-Winters No Seasonal

Original Series: ENRGLIB

Forecast Series: ENRGLISM

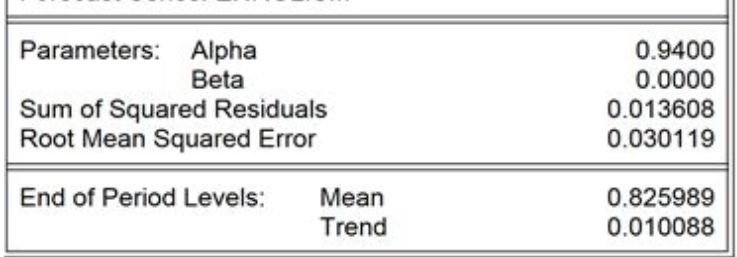

المغرب

Date: 09/15/12 Time: 21:39

Sample: 19952009

Included observations: 15

Method: Holt-Winters No Seasonal

Original Series: ENRGSUD

Forecast Series: ENRGSUSM

\begin{tabular}{|llr||}
\hline \hline Parameters: & Alpha & 0.1100 \\
\multicolumn{1}{c}{ Beta } & 1.0000 \\
Sum of Squared Residuals & 66.91801 \\
Root Mean Squared Error & 2.112156 \\
\hline \hline \multirow{2}{*}{ End of Period Levels: $\quad$ Mean } & 68.39218 \\
& $\quad$ Trend & -1.776610 \\
\hline
\end{tabular}

\section{الملحق (1). تحديد قيم المعلمات:}

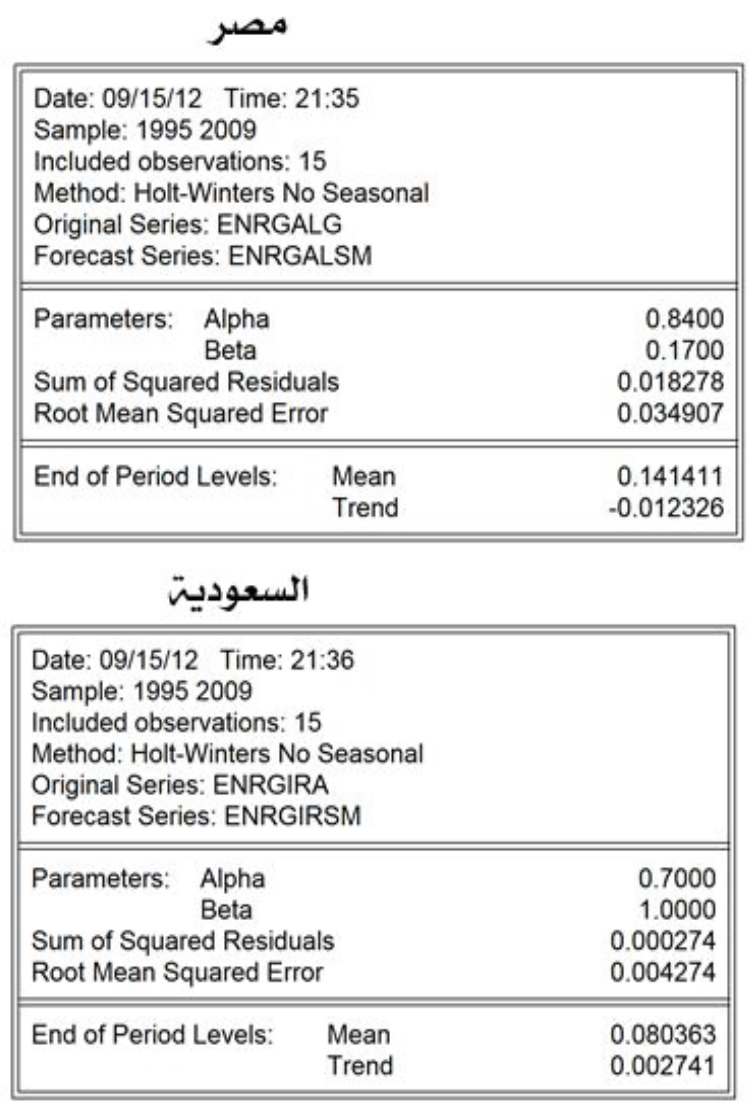

\section{ليبيا}

Date: 09/15/12 Time: 21:37

Sample: 19952009

Included observations: 15

Method: Holt-Winters No Seasonal

Original Series: ENRGLEB

Forecast Series: ENRGLESM

\begin{tabular}{|c|c|c|}
\hline 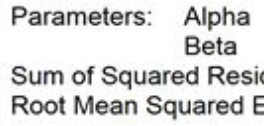 & & $\begin{array}{r}1.0000 \\
0.0000 \\
1.702548 \\
0.336902\end{array}$ \\
\hline End of Period Levels: & $\begin{array}{l}\text { Mean } \\
\text { Trend }\end{array}$ & $\begin{array}{r}1.831025 \\
-0.015671\end{array}$ \\
\hline
\end{tabular}

Date: 09/15/12 Time: 21:38

Sample: 19952009

Included observations: 15

Method: Holt-Winters No Seasonal

Original Series: ENRGMAR

Forecast Series: ENRGMASM

\begin{tabular}{|llr|}
\hline \hline Parameters: & Alpha & 0.6400 \\
& Beta & 0.0100 \\
Sum of Squared Residuals & 0.181347 \\
Root Mean Squared Error & 0.109954 \\
\hline \hline \multirow{2}{*}{ End of Period Levels: $\quad$ Mean } & 3.156407 \\
& Trend & -0.088428 \\
\hline
\end{tabular}




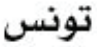

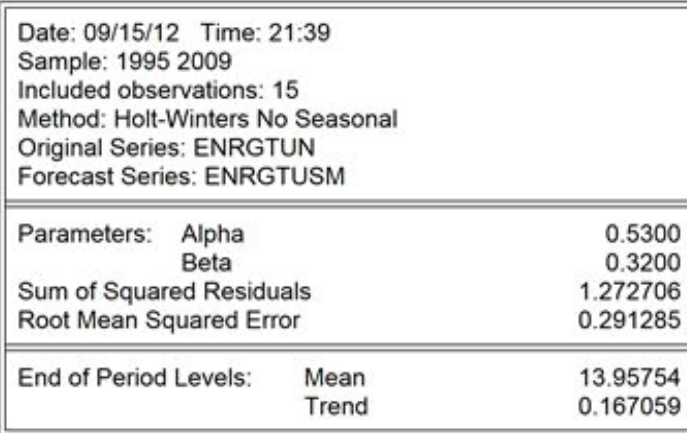

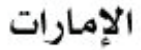

Date: 09/15/12 Time: $21: 40$

Sample: 19952009

Included observations: 15

Method: Holt-Winters No Seasonal

Original Series: ENRGYEM

Forecast Series: ENRGYESM

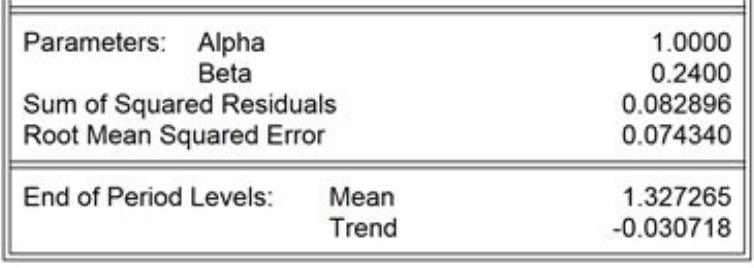

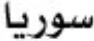

Date: 09/15/12 Time: 21:39

Sample: 19952009

Included observations: 15

Method: Holt-Winters No Seasonal

Original Series: ENRGSYR

Forecast Series: ENRGSYSM

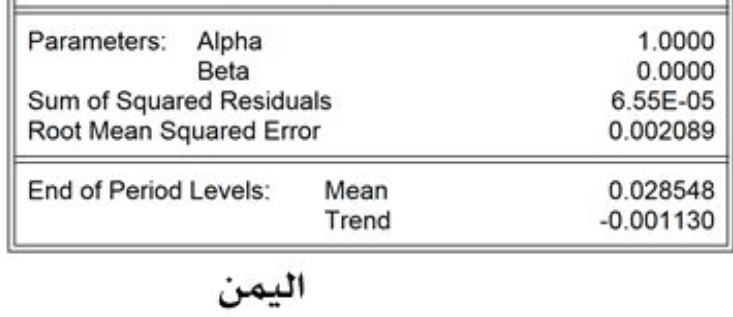

Date: 09/15/12 Time: $21: 40$

Sample: 19952009

Included observations: 15

Method: Holt-Winters No Seasonal

Original Series: ENRGUAE

Forecast Series: ENRGUASM

\begin{tabular}{|llr|}
\hline \hline Parameters: & Alpha & 0.9000 \\
& Beta & 0.1400 \\
Sum of Squared Residuals & 0.000738 \\
Root Mean Squared Error & 0.007015 \\
\hline \hline \multirow{2}{*}{ End of Period Levels: $\quad$ Mean } & 0.028090 \\
& Trend & -0.003256 \\
\hline
\end{tabular}

الملحق (2). الأشكال البيانيت للقيم التنبؤيت

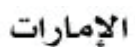

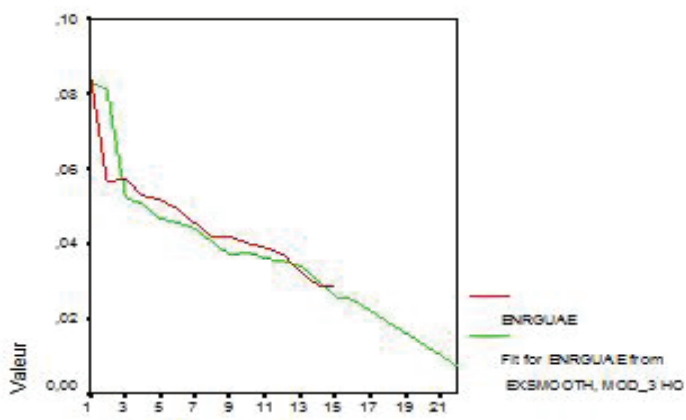

Numéro de robservation

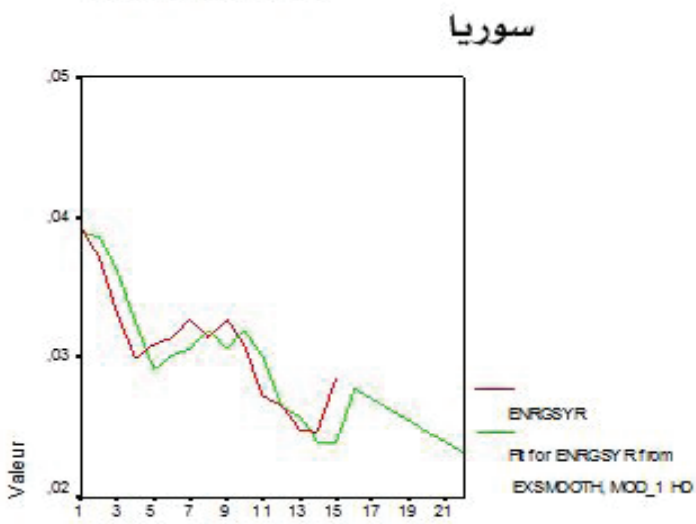

Numéro de fobservation

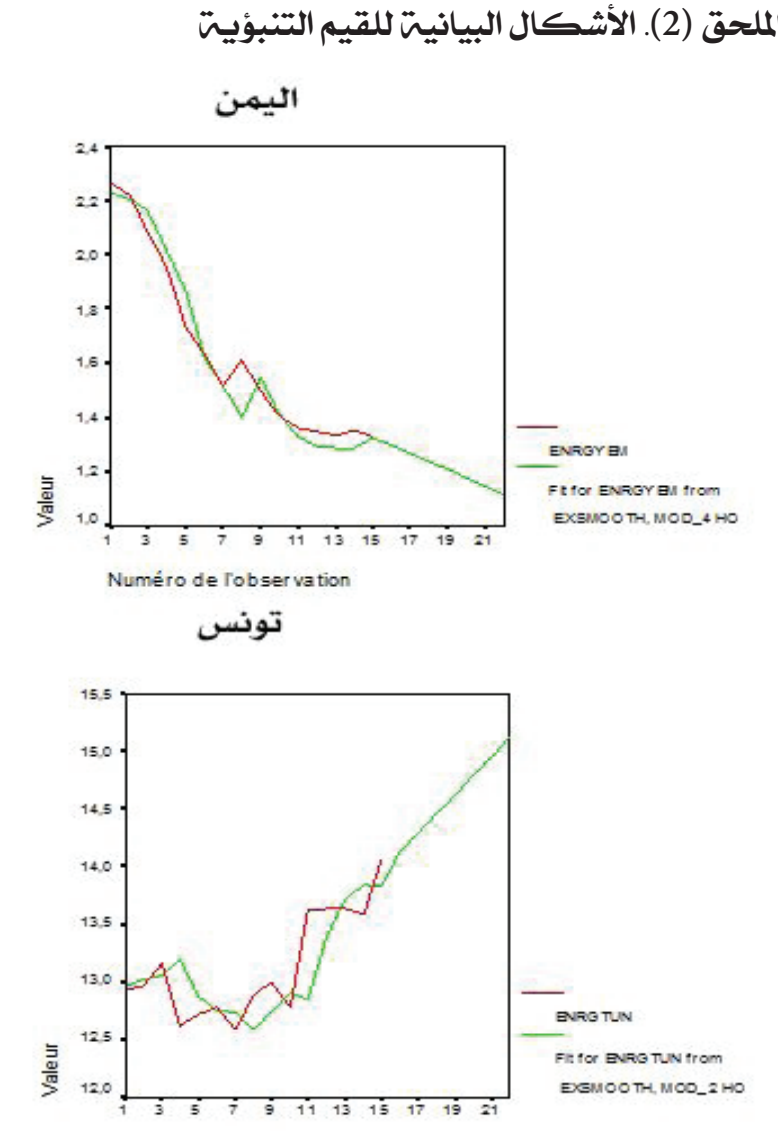

Numéro de robservation 


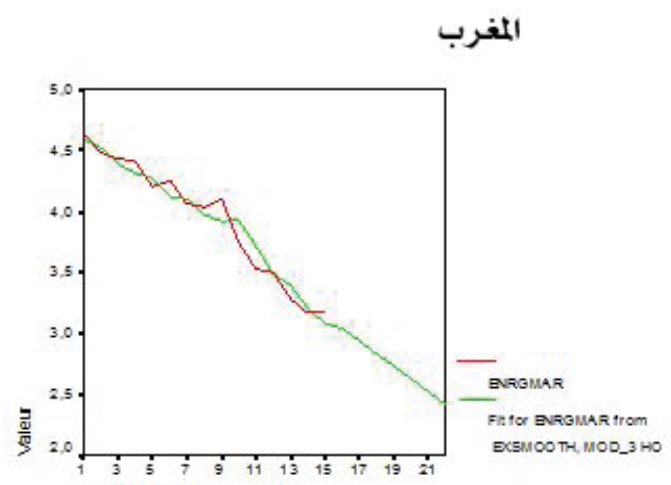

Numero de robservation

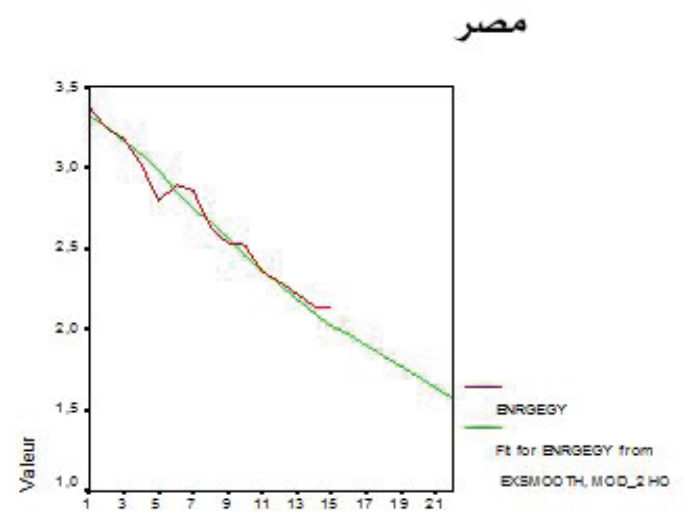

Numéro de Tobservation

\section{السعوديت}

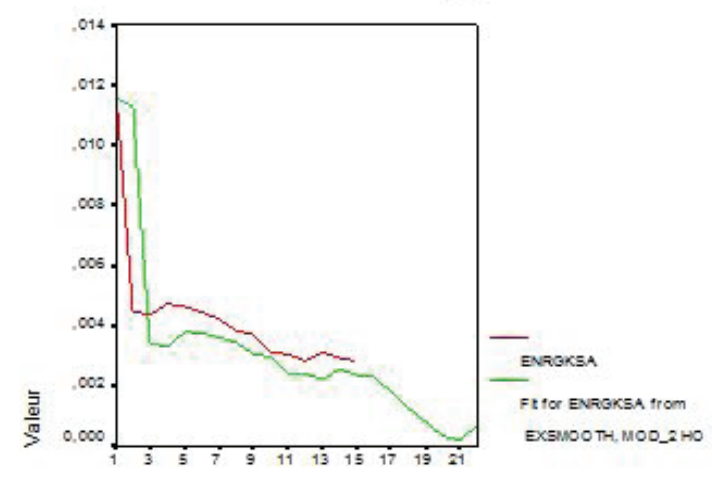

Numéro de robservation

كيبيا

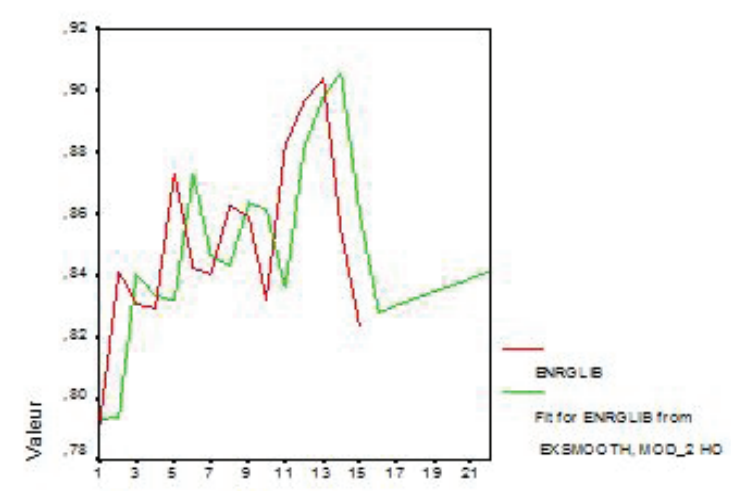

Numéro de lobservation

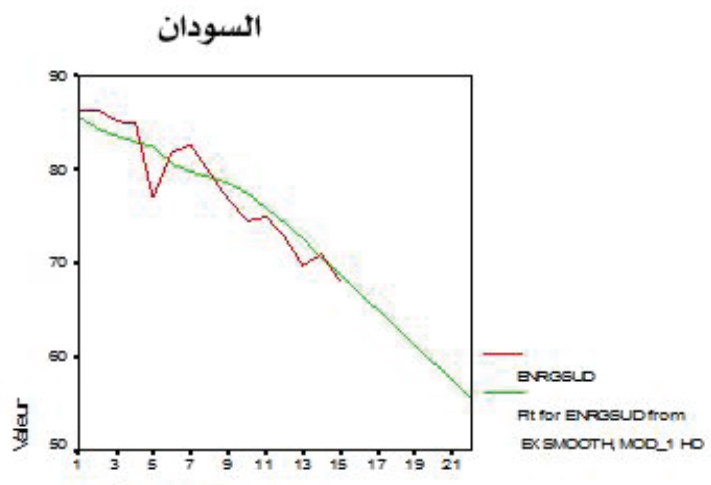

Numéro de l'observation

الجزائر

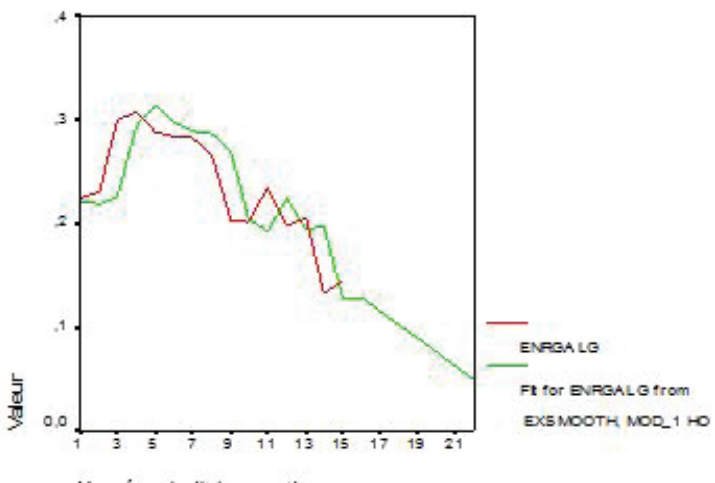

Numéro de l'obs ervation

العراق

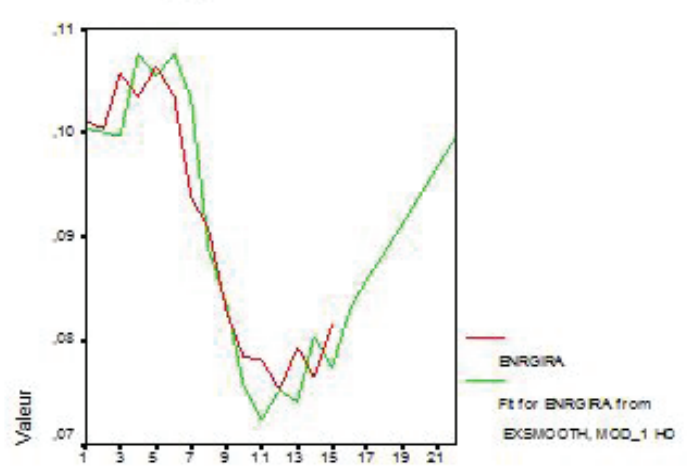

Numéro de robservation

لبنان

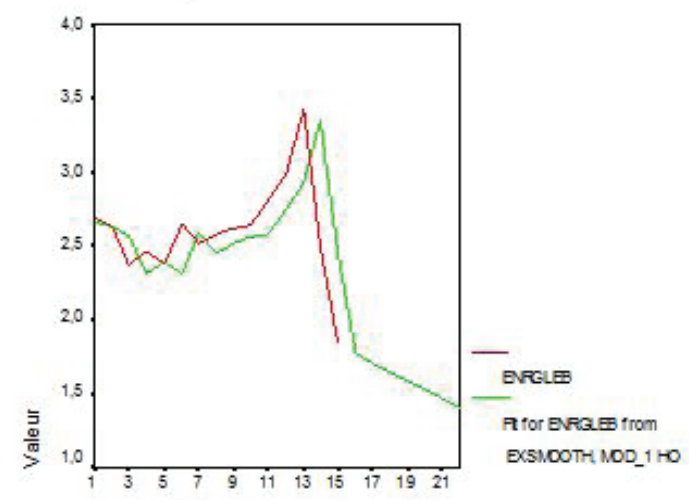

Numéro de lobservation 